\title{
Limb and Trunk Mechanisms for Balance Control during Locomotion in Quadrupeds
}

\author{
Pavel E. Musienko, ${ }^{1}$ Tatiana G. Deliagina, ${ }^{2}$ Yury P. Gerasimenko, ${ }^{1}$ Grigori N. Orlovsky, ${ }^{2}$ and Pavel V. Zelenin ${ }^{2}$ \\ ${ }^{1}$ Pavlov Institute of Physiology, 100034 St Petersburg, Russia, and ${ }^{2}$ Department of Neuroscience, Karolinska Institute, SE-17177, Stockholm, Sweden
}

In quadrupeds, the most critical aspect of postural control during locomotion is lateral stability. However, neural mechanisms underlying lateral stability are poorly understood. Here, we studied lateral stability in decerebrate cats walking on a treadmill with their hindlimbs. Two destabilizing factors were used: a brief lateral push of the cat and a sustained lateral tilt of the treadmill. It was found that the push caused considerable trunk bending and twisting, as well as changes in the stepping pattern, but did not lead to falling. Due to postural reactions, locomotion with normal body configuration was restored in a few steps. It was also found that the decerebrate cat could keep balance during locomotion on the laterally tilted treadmill. This postural adaptation was based on the transformation of the symmetrical locomotor pattern into an asymmetrical one, with different functional lengths of the right and left limbs. Then, we analyzed limb and trunk neural mechanisms contributing to postural control during locomotion. It was found that one of the limb mechanisms operates in the transfer phase and secures a standard (relative to the trunk) position for limb landing. Two other limb mechanisms operate in the stance phase; they counteract distortions of the locomotor pattern by regulating the limb stiffness. The trunk configuration mechanism controls the body shape on the basis of sensory information coming from trunk afferents. We suggest that postural reactions generated by these four mechanisms are integrated, thus forming a response of the whole system to perturbation of balance during locomotion.

Key words: decerebrate cat; limb reflexes; postural reactions; treadmill walking; trunk reflexes

\section{Introduction}

During locomotion, cats and other quadrupeds keep the dorsalside-up body orientation (basic posture). Maintenance of this orientation (i.e., the lateral stability) is of critical importance for locomotion. For example, when deprived of the ability to keep balance in their hindquarters after transection of the spinal cord, cats are not able to walk, though the basic mechanisms of stepping can function normally. However, locomotion in spinal cats becomes feasible if the lateral stability is maintained externally (Barbeau and Rossignol, 1990). Even more demanding is the maintenance of balance in walking bipeds (Hilliard et al., 2008).

Many factors can perturb the body postural configuration and balance during locomotion, and reactions to some of these factors have been studied in intact cats (for review, see Deliagina et

Received Nov. 4, 2013; revised Feb. 21, 2014; accepted March 19, 2014.

Author contributions:P.E.M., T.G.D., Y.P.G., G.N.O., and P.V.Z. designed research;P.E.M., T.G.D., Y.P.G., and P.V.Z. performed research; P.E.M., T.G.D., G.N.O., and P.V.Z. analyzed data; P.E.M., T.G.D., Y.P.G., G.N.O., and P.V.Z. wrote the paper.

This work was supported by Grants from NIH R01 NS-064964, from Christopher and Dana Reeve Foundation, from Swedish Research Council (no. 11554) to T.G.D., by Grants from Russian Foundation for Basic Research Grants (11-04-01669, 12-04-33145) and Grant of President of Russian Federation (MK-5684.2012.4) to P.E.M., by Grants from Russian Foundation for Basic Research Grants (13-04-0109a, and 13-04-12030-0FI-M) to Y.P.G., by Grant from Swedish Research Council (no. 21076) to P.V.Z. We thank Dr Brita Robertson for valuable comments on the paper, Medynja Kutueva for the excellent technical assistance during surgeries and experiments, as well as for animal care, and Vyacheslav Kilimnik and Alexandr Savochin for engineering support and help in the development of the experimental setup.

The authors declare no competing financial interests.

Correspondence should be addressed to Dr P. V. Zelenin, Department of Neuroscience, Karolinska Institute, SE-17177, Stockholm, Sweden. E-mail: Pavel.Zelenin@ki.se

DOI:10.1523/JNEUROSCI.4663-13.2014

Copyright $\odot 2014$ the authors $\quad 0270-6474 / 14 / 345704-13 \$ 15.00 / 0$ al., 2012). These studies have shown that the walking cat is able to compensate for body displacement in the transverse plane caused by a brief lateral push applied to the trunk (Karayannidou et al., 2009a). The cat is also able to walk and maintain balance on the unstable support, e.g., during lateral translation or dropping of the supporting platform (Marple-Horvat et al., 1993; Misiaszek, 2006). The cat can also adjust its stepping movements to environmental conditions when walking along an inclined supporting surface (Matsuyama and Drew, 2000; Karayannidou et al., 2009b). However, the neural mechanisms underlying postural reactions to these destabilizing factors remain to be identified. In particular, it is not clear which neural structures are involved in postural reactions to different perturbations of balance during locomotion.

The analysis of balance control during locomotion is a difficult problem because of a great number of motor centers and control mechanisms operating simultaneously. One of possible approaches is the use of reduced preparations, in which the number of control mechanisms is decreased. Recently, it was demonstrated that the decerebrate cat is able to maintain equilibrium of its hindquarters when walking on the horizontal treadmill (Musienko et al., 2012a). In the first part of the present study, we addressed the question if the decerebrate cat during locomotion can compensate for various destabilizing factors? We have found that the decerebrate cat can counteract such destabilizing factors as a lateral push and a lateral tilt of the treadmill, and these postural reactions are similar to those in intact cats (Karayannidou et al., 2009a,b). We conclude that the basic balance mechanisms reside below the decerebration level, and that the decerebrate 
Type 1 experiments: pelvis free $(\mathrm{N}=6)$

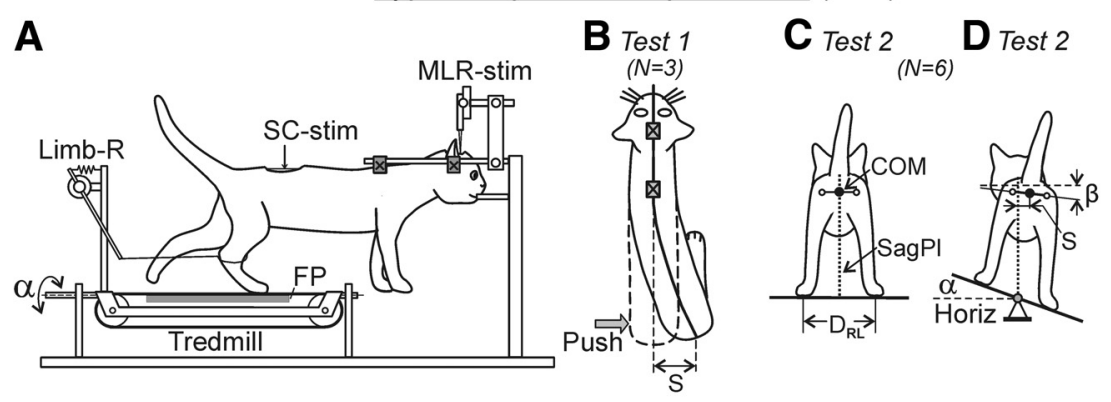

Type 2 experiments: pelvis fixed $(\mathrm{N}=8)$

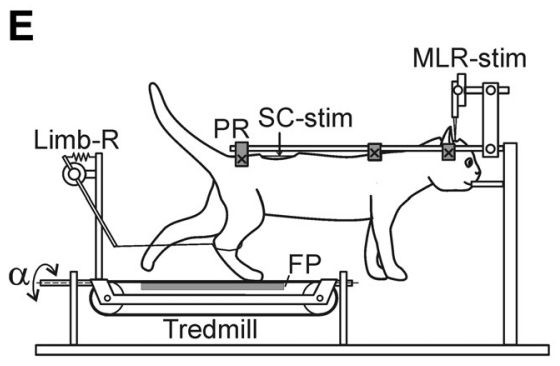

F Tests 3,4

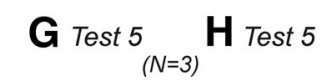

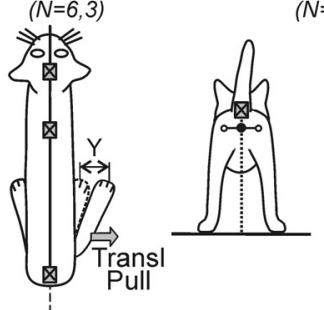

Type 3 experiments: pelvis suspended $(\mathrm{N}=4)$

I

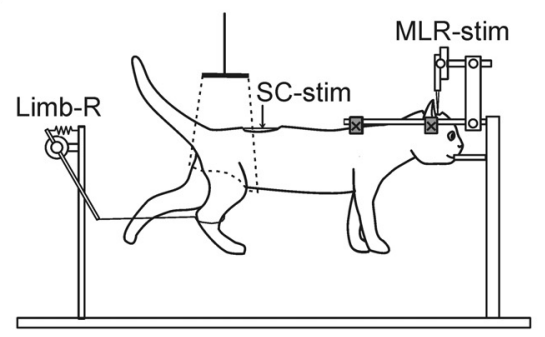

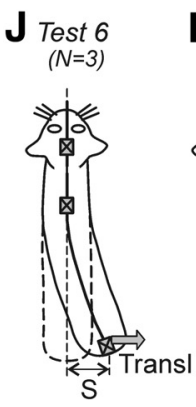

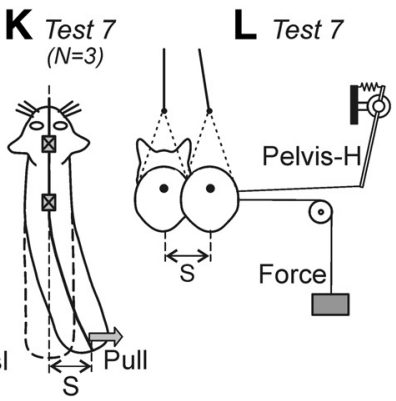

Figure 1. Design of three types of experiments. $A, E, I$, In all experiments, the decerebrate cat was fixed in a rigid frame (points of fixation are indicated by crosses). Walking of the hindlimbs was evoked by stimulation of the mesencephalic locomotor region (MLR-stim) or the spinal cord (SC-stim). $\boldsymbol{A}-\boldsymbol{D}$, Type 1 experiments. The hindlimbs were positioned on the moving belt of the treadmill, the pelvis was not fixed, and balance in the hindquarters was maintained due to the postural neural mechanisms of the cat. Postural reactions were evoked by the lateral push of the pelvis (Test 1, B) or the lateral treadmill tilt $\alpha$ (Test 2, Cand $\boldsymbol{D}$ ). $\boldsymbol{E}$ - $\boldsymbol{H}$, Type 2 experiments. The hindlimbs were positioned on the moving belt of the treadmill, the pelvis was fixed by the pelvis restraint (PR), and no balance control was required. Reactions of individual limbs to destabilizing factors, i.e., to the lateral translation and the lateral pull (Tests 3,$4 ; \boldsymbol{F})$, as well as to the lateral tilt (Test $5 ; \boldsymbol{G}, \boldsymbol{H}$ ) were recorded. $\boldsymbol{I}-\boldsymbol{L}$, Type 3 experiments. The pelvis was supported by the pelvis restraint that could be moved horizontally to the right and to the left $(J)$, or it was suspended on a long string $(\boldsymbol{I}, \boldsymbol{K}, \boldsymbol{L})$. The treadmill was removed. A lateral translation of the pelvis was performed by moving PR (Test $6 ; \boldsymbol{J})$ or by applying a constant lateral force, (Test $7 ; \boldsymbol{K}, \boldsymbol{L})$ during air stepping. The anterior-posterior position of each limb was recorded by a sensor; only one sensor (Limb-R) is shown in $\boldsymbol{A}, \boldsymbol{E}, \boldsymbol{I}$. The contact force under each limb was measured by a force plate (FP). The lateral displacement of the pelvis $(S)$ relative to the sagittal plane ( $\mathrm{SagPl} ; \boldsymbol{C}, \boldsymbol{D}$, dotted line) was recorded by means of a mechanical sensor. The lateral displacement of the limb $(\mathrm{Y})$ in Tests 3, $4(\boldsymbol{F})$ was recorded by another sensor. The number of animals used in each of three types of experiments, as well as the number of animals subjected to each of seven tests is indicated in parentheses.

preparation can be used for studying the balance control during locomotion.

In the second part of the present study, we tested the hypothesis that a number of rather autonomous neural mechanisms (each with its specific function, e.g., stabilization of the lateral limb position, stabilization of the trunk shape) participate in the control of balance during locomotion. We revealed four such mechanisms, and then suggested their contribution to the postural reactions of the whole system (described in the first part of this paper).

A brief account of this study was published in abstract form (Zelenin et al., 2013).

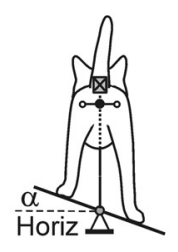

\section{Materials and Methods}

Subjects. Experiments were performed on 11 adult cats of either sex (weighing 2.1-2.9 kg). All procedures were conducted according to the European Community Council Directive (November, 1986; 86/609/EEC) in accordance with a protocol approved by the Animal Care Committee of the Pavlov Institute of Physiology, St Petersburg, Russia, and followed the guidelines of the National Institute of Health Guide for the Care and Use of Laboratory Animals.

Surgical procedures. The surgical procedures were similar to those used in the previous study (Musienko et al., 2012b). The cats were deeply anesthetized with a mixture of xylazine ( $1 \mathrm{mg} /$ $\mathrm{kg}$, i.m.) and ketamine $(40 \mathrm{mg} / \mathrm{kg}$, i.m.), and supplemented by $30-50 \%$ of the initial dose if necessary. The level of anesthesia was monitored based on applying pressure to the paw (to detect limb withdrawal), as well as by checking the size and reactivity of the pupils. The trachea was cannulated and the carotid arteries were ligated. The animal was decerebrated at the precollicular-postmammilar level. A laminectomy was performed at the L5 level. Bipolar EMG electrodes $(0.2 \mathrm{~mm}$ flexible stainless steel Teflon-insulated wires) were implanted bilaterally into gastrocnemius lateralis (Gast, ankle extensor), tibialis anterior (Tib, ankle flexor), adductor femoris (Add, hip extensor and adductor), and gluteus medius (Glut, hip extensor and abductor) as described previously (Gerasimenko et al., 2009). Our reason for recording these particular muscles was that Gast and Tib take part in the longitudinal component of step, whereas Glut and Add also take part in the lateral component of step. Some other hindlimb muscles: gracilis (Grac, hip extensor and adductor) and vastus medialis (Vast, knee extensor), as well as sartorius (hip flexor), iliopsoas (Iliop, hip flexor), rectus femoris (knee extensor), and caudofemoralis (hip extensor) were recorded in one to three experiments. In three experiments, EMGs of trunk muscles (erector spinae and multifidus) were recorded at the L5-L6 vertebral level. Anesthesia was discontinued after the surgical procedures, and the experiments were initiated $2-3 \mathrm{~h}$ thereafter.

During the experiment, the rectal temperature and mean blood pressure of the animal were continuously monitored and were kept at $37 \pm 0.5^{\circ} \mathrm{C}$ and $>80 \mathrm{mmHg}$.

Experimental design. The experimental design (Fig. 1) was in many respects similar to that used in the previous study (Musienko et al., 2012b). Three types of experiments were performed. In Type 1 experiments (for studying the capacity of the walking decerebrate cat to compensate for destabilizing factors) the head and the rostral part of the vertebral column (at Th3) were fixed in a rigid frame, whereas the caudal part of the vertebral column and the pelvis were unrestrained (Fig. 1A). The forelimbs had no support, whereas the hindlimbs were positioned on the treadmill with two separate belts (left and right) moving at the same speed $(0.5 \mathrm{~m} / \mathrm{s})$ and below referred to as the "treadmill belt." The belt was moving backward in relation to the animal. In these experiments, the balance in the hindquarters during locomotion was maintained due to the activity of neural postural mechanisms of the cat's hindlimbs and trunk. 
In Type 2 experiments (devoted to the analysis of the limb postural mechanisms), not only the head and rostral part of the vertebral column, but also the pelvis were fixed in a rigid frame (Fig. 1E). The distance between the treadmill belt and the fixed pelvis was $21-25 \mathrm{~cm}$ (depending on the animal size), which determined a hemi-flexed limb configuration in the middle of stance typical for walking.

In Type 3 experiments (devoted to the analysis of the trunk postural mechanisms), the hindquarters of the cat with the unrestrained pelvis were suspended in a hammock, the treadmill was removed, and the hindlimbs performed air-stepping (Fig. 1I).

To examine the balance control during locomotion and to reveal the underlying neural mechanisms, seven tests were carried out. In Type 1 experiments, two tests were performed. In Test 1 (Fig. 1B), brief pulses of force (pushes) were applied in the latero-medial direction in the hip joint area. The pushes were applied in different phases of the step cycle, once every 5-10 steps. They were performed manually, and the cases with a push-caused pelvis displacement by $4-10 \mathrm{~cm}$ were selected for the analysis. The cases with too forceful pushes, which caused a lateral displacement of the supporting limb, were excluded from further analysis. In Test 2 (Fig. $1 C, D$ ), the treadmill under the cat was periodically tilted in the transverse plane between the two stationary positions $\left( \pm 20^{\circ}\right.$ in relation to the horizon; angle $\alpha$ in Fig. $1 A, D$ ). A transition from one stationary position to the other lasted for 2-3 s, and each position was maintained for $8-12$ s (see Fig. $5 A$ ).

In Type 2 experiments, three tests were carried out. In Test 3, we performed a rapid lateral (or medial) translation of the supporting surface under one of the limbs. For this purpose, a small plate $(10 \times 20 \mathrm{~cm})$ was positioned on the corresponding part of the treadmill belt, with its longitudinal axis perpendicular to the treadmill velocity vector. When the limb stepped on this plate, it was quickly translated to a new, more lateral or medial position (Figs. $1 F$, Transl; $6 \mathrm{~A}$ ); the limb continued to perform stance at this new lateral position. The magnitude of the lateral and medial translations were $4-12$ and $4-8 \mathrm{~cm}$, respectively. In Test 4 , we applied a constant lateral (or medial) force $(0.15-0.2 \mathrm{~kg}$ ) to the distal part of the limb by means of the elastic string of $20 \mathrm{~cm}$ in length (Figs. $1 F$, Pull; 7A). The applied force did not evoke displacement of the limb during stance, but caused substantial lateral or medial deviation of the limb during swing. In Test 5 , the treadmill under the cat was periodically tilted (by $\pm 20^{\circ}$ ) in the transverse plane (Fig. $1 H, \alpha$ ) with the same temporal pattern as in Test 2. Because in Type 2 experiments the pelvis was rigidly fixed, the tilt caused loading of the limb whose support was moving up, and unloading of the opposite limb.

In Type 3 experiments, two tests were performed. In Test 6, lateral trunk bending was caused by a lateral translation of the pelvis restraint (Fig. 1 J, Transl) during air stepping (Musienko et al., 2012b). In Test 7, we studied how the activation of locomotor mechanisms affected the trunk configuration. For this purpose we caused a lateral trunk bending by applying a constant lateral force to the pelvis (Fig. $1 K, L$, Pull and Force, respectively) and then evoked locomotion.

The number of animals $(N)$ and trails $(n)$ for different experimental conditions are indicated either in the figure legends or in the main text. In addition, in Figure 1 the number of animals used in different types of experiments and in different tests is indicated. Usually each animal was used in several tests.

In the decerebrate cat, locomotion can be evoked from a number of different sources (for review, see Orlovsky et al., 1999; Rossignol et al., 2006), i.e., by electrical stimulation of the mesencephalic locomotor region (MLR) (Shik et al., 1966; Shik and Orlovsky, 1976; Jordan, 1986; Garcia-Rill and Skinner, 1987a,b) and by epidural electrical stimulation of the spinal cord (SC; Iwahara et al., 1992; Musienko et al., 2007, 2012b). Recently it was shown that the locomotor mechanisms activated from these two sources are partly different (Musienko et al., 2012b). To find out if this difference is critical for the operation of the limb and trunk mechanisms of balance control during locomotion, we evoked locomotion by stimulating either the MLR or SC. For MLR stimulation, the bipolar electrode (two $150 \mu \mathrm{m}$ wires isolated except for the tips and separated by $0.5 \mathrm{~mm}$ ) was inserted into the brainstem area (HorsleyClark coordinates $\mathrm{P} 2, \mathrm{R} / \mathrm{L} 4, \mathrm{H} 0$ ) by means of a micromanipulator (Fig. $1 A, E, I ;$ MLR-stim). We used the following parameters of stimulation: frequency, 30 pulses per second (pps); pulse duration, 0.5-1 ms; current, $50-200 \mu$ A. For epidural SC stimulation, a ball electrode $(\mathrm{d}=0.5 \mathrm{~mm})$ was positioned on the dura mater in the middle of the dorsal surface of the spinal cord at the L5 level (Fig. 1 A, E,I; SC-stim). We used the following parameters of stimulation: frequency, $5 \mathrm{pps}$; pulse duration, $0.2-0.5$ $\mathrm{ms}$; current, $100-300 \mu \mathrm{A}$. In some animals locomotion was initiated by stimulation of MLR and by stimulation of SC.

In each test, the rear view and the side view of the walking cat were recorded by two video cameras ( 25 or 50 frames/s). In addition, in all tests we recorded the anterior-posterior locomotor limb movements (by means of two mechanical sensors, one of which is shown in Fig. 1 A,E, I; Limb-R), as well as the vertical forces developed by each of the limbs (by means of two force plates positioned under the left and right parts of the moving belt; Fig. $1 A, E$; FP). In Tests $1,2,6$, and 7 , the lateral pelvis movements in the horizontal plane (Fig. $1 B, D, J-L ; S$ ) were recorded by a sensor similar to Limb-R sensor (Fig. $1 L$; Pelvis-H). Such sensor was also used for monitoring the lateral foot position in Tests 3 and 4 (Fig. $1 F$; $\mathrm{Y})$. Mechanical sensor consisted of a variable resistor whose axis was rotated by means of a long lever; the latter was attached to the limb or pelvis by means of long string. In Tests 2 and 5 , the treadmill tilt (Fig. $1 A$, $D, H$; angle $\alpha$ ) was monitored by a variable resistor whose axis was rotated by the tilting platform. Video recording, EMG recording, and recording with mechanical sensors were synchronized.

Data analysis. The signals from the EMG electrodes and from the position sensors were amplified, digitized with a sampling frequency of 5 $\mathrm{kHz}$ (EMGs) and $1 \mathrm{kHz}$ (sensors), and recorded on a computer disk using the data-acquisition and analysis software (Power-1401/Spike2, Cambridge Electronic Design). The EMG signals were either rectified and smoothed (time constant, 50-100 ms) or averaged ( $>5-20$ steps) for each of the 100 bins of the step cycle that was normalized to 1.0. The beginning of the swing phase was taken for the cycle onset.

The video recordings were analyzed frame-by-frame. From the rear view, an approximate position of the center of mass (COM) of the hindquarters (the anus coordinates), the COM projection on the horizontal plane, the step width, as well as the position of two markers on lateral parts of the pelvic bones were estimated. The latter were used to calculate the pelvis tilt angle (Fig. $1 D ; \beta$ ). From the rear view and/or from the side view it was easy to establish whether the foot was on the ground (i.e., if the limb was in the stance phase of its step cycle) or the foot was lifted (i.e., the limb was in the transfer phase of the cycle). During the stance phase, the vertical foot coordinate $Z=0$ and the foot trajectory could be entirely characterized by two coordinates, $X$ and $Y$ (see Figs. 6, 7). A coordinate grid (that compensated for distortions of perspective) was applied to the image of the treadmill area. Using this grid, foot coordinates were measured in sequential frames. During the swing phase, the foot was lifted above the ground, but its vertical coordinate $Z$ could not be precisely measured with our methods. Therefore, the foot trajectory during swing could be characterized only roughly in $X / Y$ coordinates.

All quantitative data in this study are presented as mean \pm SD or SE. Student's $t$ test was used to characterize the statistical significance when comparing different means; the significance level was set at $p=0.05$.

\section{Results}

\section{Capacity of decerebrate cat to compensate for} destabilizing factors

\section{Unperturbed locomotion}

Figure $2 A$ illustrates Type 1 experiment; i.e., unperturbed locomotion with the free pelvis, which was evoked by MLR stimulation in one of the cats. The alternating stepping movements and contact forces of the right and left limbs were rather uniform. During locomotion, the pelvis exhibited horizontal step-related oscillations (S) with a peak-to-peak value of $\sim 7 \mathrm{~cm}$. In addition to these oscillations, video recording of the rear view revealed vertical oscillations with a much smaller peak-to-peak value $(\sim 2$ $\mathrm{cm}$, not illustrated)

Figure $2 B$ shows configuration of the hindquarters in the transverse plane (based on video recording) observed at different time points (1-7). Positions of these time points relative to the 


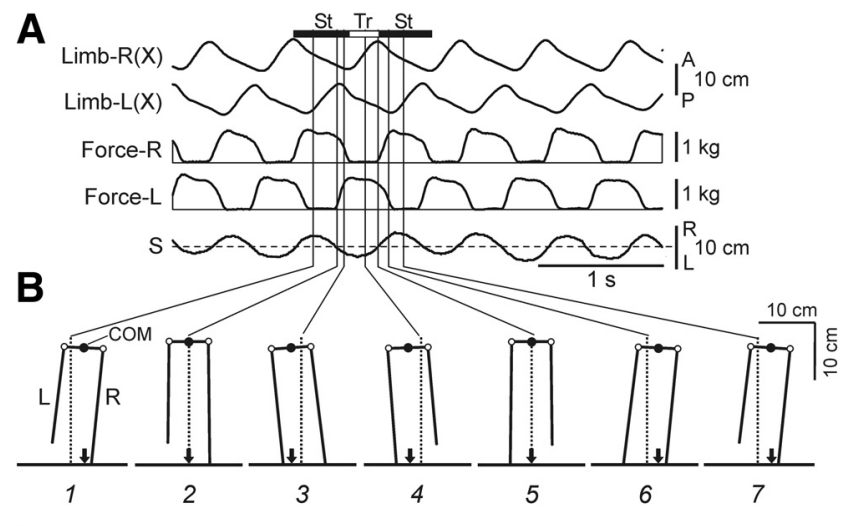

C

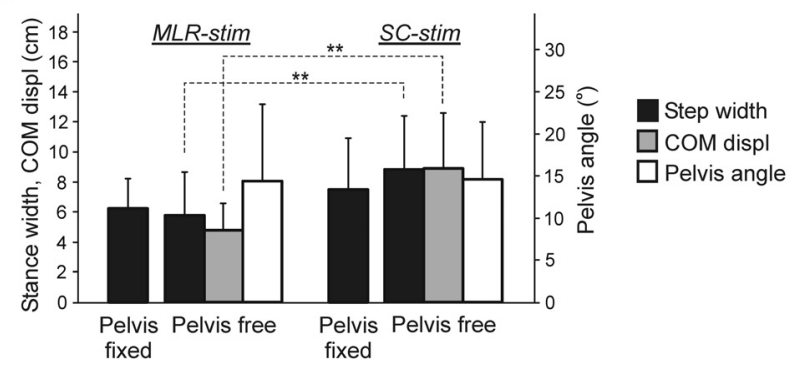

Figure 2. Unperturbed locomotion on the horizontal surface (Type 1 experiments). $A$, Anteroposterior movements and vertical forces of the right $(R)$ and left $(L)$ limb, as well as horizontal pelvis oscillations $(S)$ during locomotiom with unrestrained pelvis. $\boldsymbol{B}$, Configuration of the hindquarters (rear view) in different phases of the step cycle (1-7). These time points in the St and Tr phases of the right limb are indicated in $A$. Designations: empty circles, hip joints; black arrow, projection of COM. $C$, The mean value ( $\pm S D$ ) of the step width with pelvis fixed and pelvis free, the lateral peak-to-peak displacements of $\mathrm{COM}$, and the pelvis peak-to-peak rotations (Pelvis angle) during MLR-evoked and SC-evoked locomotion. For MLR-evoked locomotion: step width with pelvis fixed $(N=5, n=105)$, step width with pelvis free $(N=4, n=81)$, COM displ ( $N=3, n=57)$, Pelvis angle $(N=3, n=81)$. For SC-evoked locomotion: step width with pelvis fixed ( $N=6, n=98)$, step width with pelvis free $(N=4, n=85)$, COM displ ( $N=$ $4, n=85)$, and Pelvis angle $(N=4, n=89)$. Indication of significance level: ${ }^{* *} p<0.01$.

stance (St) and transfer (Tr) phases of the right limb are indicated in Figure $2 A$. In this particular cat, the stance width (Fig. $1 C ; \mathrm{D}_{\mathrm{RL}}$ ) was close to the width of the pelvis $(\sim 7 \mathrm{~cm})$, and the axes of the right and left limbs (i.e., the lines connecting the foot and hip joints) were almost perpendicular to the pelvis and parallel to each other. From Figure $2 B$, one can see that the pelvis exhibited periodical tilts to the right (panels $1,6,7$ ) and to the left (panels 3,4 ) due to its rotation around the longitudinal body axis (Fig. $1 D$; angle $\beta$ ). The peak-to-peak value of these rotatory oscillations was rather small (Fig. 2C, Pelvis angle).

From Figure $2 B$ one can also see that, during locomotion, the COM of the hindquarters (estimated by the anus position; Fig. $1 C$ ), and its projection on the supporting surface (indicated by an arrow) exhibited step-related lateral oscillations. These oscillations reflect the anticipatory postural adjustment, which causes the COM to be propelled toward the stance-limb side before the lifting of the swing foot (McIlroy and Maki, 1999). From Figure $2 B$ it is seen that the COM projection always occurred within the step width (also termed "the support area"), which is an important condition for postural stability (Hof et al., 2010).

The main characteristics of locomotion described above for one cat were observed in other cats as well. Comparison of these characteristics in the cats with MLR and SC-evoked locomotion is presented in Figure $2 C$. One can see that the step width and the peak-to-peak lateral COM displacements (COM displ) were somewhat larger during SC-evoked locomotion than during
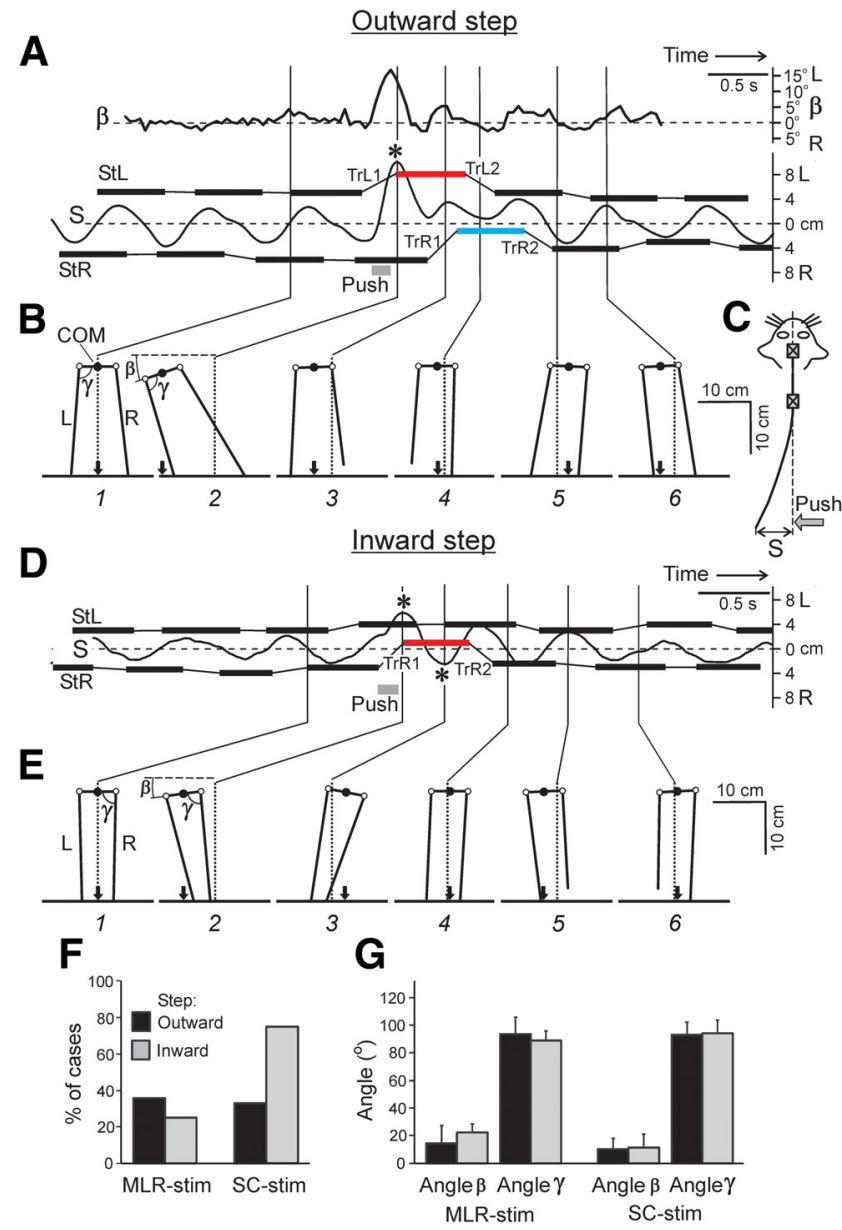

Figure 3. Effects of lateral push (Test 1). A-C, A leftward push during the transfer phase of the left limb causing outward step. $\boldsymbol{D}, \boldsymbol{E}, \mathrm{A}$ leftward push during the transfer phase of the right limb causing inward step. Position of the right and left foot during stance (StR and StL, based on video data), and a distance $(S)$ of the pelvis from the sagittal plane (interrupted line) are shown in $A$ and $\boldsymbol{D}$ as a function of time (data from the mechanical sensor). Asterisks indicate the points where COM projection appeared outside of the step width (also based on video data). $\operatorname{TrL} 1, \operatorname{TrL2}$, TrR1, and TrR2 are sequential transfer phases of $\mathrm{L}$ - and R-limbs during and after the push. $\boldsymbol{B}, \boldsymbol{E}$, Configuration of the hindquarters (rear view) at different time points (1-6). The angle between pelvis and horizon $(\beta)$, as well as between pelvis and $\operatorname{limb}(\gamma)$ at the moment of touchdown are indicated. Other designations as in Figure 2. $F$, Proportion of cases when COM projection occurred outside the step width under different conditions. $\boldsymbol{G}$, Angles $\beta$ and $\gamma($ mean \pm SD) in cats with MLR stimulation and $\mathrm{SC}$ stimulation. $(\boldsymbol{F}, \boldsymbol{G}$ : outward and inward steps during MLRstimulation $N=2,1$ and $n=14,9$, respectively. Outward and inward steps during SCstimulation $N=2,2$ and $n=12,8$, respectively).

MLR-evoked locomotion (the difference was statistically significant), while the amplitudes of pelvic rotation were similar. No difference was found between the step width under pelvis free and pelvis fixed conditions.

\section{Reactions to lateral push}

These experiments were performed on cats $(N=6)$ with unrestrained pelvis (Fig. $1 B$, Test 1). We have found that these cats were able to keep balance during locomotion despite strong lateral pushes that displaced their pelvis by $5-10 \mathrm{~cm}$. Two types of reactions were observed depending on the phase and direction of push, i.e., the outward and inward steps.

\section{Outward step}

An example of this type of reaction is shown in Figure $3 A$. The horizontal bars (StL and StR) indicate the lateral foot position (i.e., the distance from the sagittal plane of the fixed forequarters) 
for the left and right limbs in sequential stance phases, plotted against time. The Tr phases are indicated by thin lines connecting the bars. Trace $\beta$ shows the pelvis tilt, whereas trace $S$ shows the lateral pelvis position (Fig. $1 B$ ).

The leftward push, which was applied to the pelvis in the transfer phase of the contralateral (left) limb, caused a large leftward displacement of the pelvis; at its peak, the COM projection appeared outside the stance area (this event is marked by an asterisk in Fig. $3 A$ ). Because the rostral part of the vertebral column was rigidly fixed, the push-caused leftward displacement of the pelvis $(\mathrm{S})$ was accompanied by a lateral trunk bending (Fig. $3 C$ ). The push also evoked a tilt of the pelvis (Fig. $3 A ; \beta$ ) due to its rotation around the longitudinal body axis; the tilt was associated with trunk twisting.

This leftward push caused significant changes in the pattern of limb stepping. The left limb was transferred (TrL1) and landed much more laterally than during ordinary steps, which resulted in a more lateral foot trajectory in the following stance (red bar in Fig. 3A). During the next transfer phase (TrL2), a close-tonormal lateral position of the limb was restored. In the right limb, the reaction to push was considerably delayed: it appeared only in the next step ( $\operatorname{TrR} 1$ ), and the limb was transferred to a much more medial position than during ordinary steps, which resulted in a more medial foot trajectory in the following stance (Fig. $3 \mathrm{~A}$, blue bar). During the transfer phase (TrR2) of the next step, the right limb returned to the close-to-normal lateral position in stance. However, in most cases with outward steps, the lateral position of stepping limb on the side of push application was not changed (Fig. 4A, B).

There are two possible reasons for the more lateral landing of the left limb: (1) the lateral movement of the limb together with the pelvis, and (2) the lateral movement of the limb relative to the pelvis (due to the limb abduction). To estimate the contribution of these two factors, in Figure $3 B$ we compared the hindquarters configuration in an ordinary step (panel 1) and in a perturbed step, at the maximal push-caused pelvis displacement (panel 2). One can see that the angle $\gamma$ between the pelvis and the left limb (at the moment of limb touching down) in the two cases was almost the same $\left(\sim 90^{\circ}\right)$. This result suggests that the lateral displacement of the pelvis (rather than limb abduction) was the main reason for the lateral limb landing in the perturbed step.

The outward step was observed in all cats if the push was applied in the transfer phase of the limb contralateral to side of push application, both with MLR stimulation and with SC stimulation. Figure $4 A, B$ shows the mean values ( \pm SE) characterizing the lateral component of step in sequential cycles for both hindlimbs during MLR- and SC-evoked locomotion (Fig. $4 A$ and $B$, respectively). Under both conditions, in the cycle with push (cycle 0 ), the contralateral (effective) limb performed a large outward step, and in the cycle following the affected cycle (cycle 1), it performed a large inward step to return to the normal mediolateral position. The lateral component of steps in the limb ipsilateral to the side of push application was practically unchanged.

\section{Inward step}

An example of this type of reaction is shown in Figure $3 D$, E. The leftward push was applied to the pelvis in the transfer phase of the right limb. The push caused a large lateral displacement of the pelvis and its rotation. At the peak displacement, the COM projection appeared outside of the stance area (marked by an asterisk), as described above for outward steps. The push also caused a large inward step of the right limb $(\operatorname{TrR} 1)$ followed by a medially positioned stance (red bar). The limb returned to the normal

\section{Outward step}

A MLR-stim

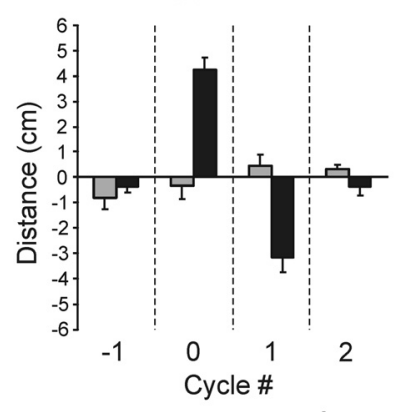

B SC-stim

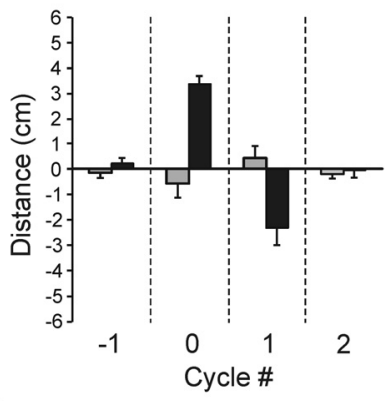

Inward step

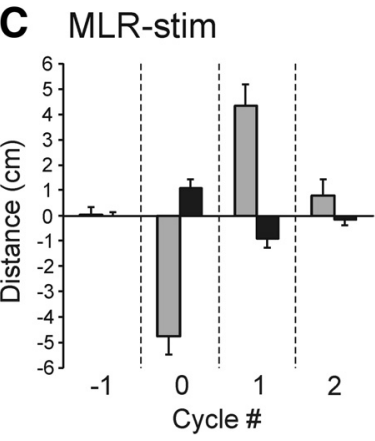

D SC-stim

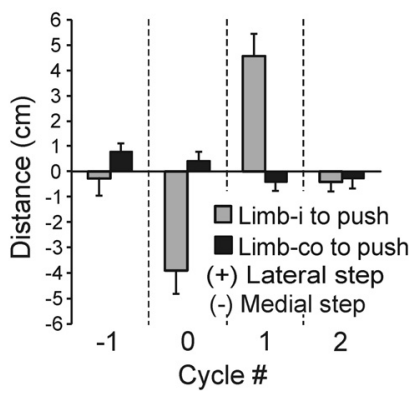

Figure 4. Summary of changes in locomotor pattern caused by pushes (Test 1). Outward steps $(\boldsymbol{A}, \boldsymbol{B})$ and inward steps $(\boldsymbol{C}, \boldsymbol{D})$ caused by a push were averaged separately for MLR-evoked locomotion $(\boldsymbol{A}, \boldsymbol{C})$ and $S$-evoked locomotion $(\boldsymbol{B}, \boldsymbol{D})$. Bars indicate mean values ( $\pm \mathrm{SE}$ ) of the lateral component of step in sequential cycles. Positive and negative values correspond to outward an inward displacement of the limb, respectively. Designation of cycles: $(-1)$, the cycle before push; (0), the cycle including push; (1), the cycle next to the affected cycle; (2), the cycle next to (1). $\boldsymbol{A}, N=2, n=16 ; \boldsymbol{B}, N=2, n=12 ; \boldsymbol{C}, N=1, n=11 ; \boldsymbol{D}, N=2, n=12$ ).

lateral position in the next step ( $\operatorname{TrR} 2)$. The lateral component of steps in the left limb was practically unchanged. Figure $3 E$ shows the hindquarters configuration (at the moment of touch down) in the ordinary step (panel 1) and in the perturbed step (panel 2). One can see that the angle between the pelvis and the limb $(\gamma)$ in the two cases was almost the same $\left(\sim 90^{\circ}\right)$, as described above for outward steps.

The inward step was observed in all cats if the push was applied in the transfer phase of the limb ipsilateral to push, both with MLR stimulation (Fig. 4C) and with SC stimulation (Fig. 4D).

These experiments have demonstrated that all tested decerebrate cats $(N=6)$ well compensated for lateral pushes; usually only a few cycles were needed to restore normal stepping. Even strong pushes, which caused a displacement of the COM projection outside of the stance area, did not lead to falling. Such cases were observed in all cats, both in outward and inward steps, as well as during MLR and SC-evoked locomotion (Fig. 3F). Apparently, to prevent a sideward falling of the hindquarters in these cases and to return the COM projection into the stance area, an external force has to be applied to the pelvis and caudal trunk, in the direction opposite to the push. We suggest that this force is generated due to the reflex activation of back muscles (see Trunk configuration mechanism).

These experiments have also shown that, despite a considerable push-caused lateral movement of the pelvis (up to $10 \mathrm{~cm}$ ) and its rotation (up to $20^{\circ}$ ), the limb performing outward or inward step, at the end of swing was situated at a constant angle 


\section{A}
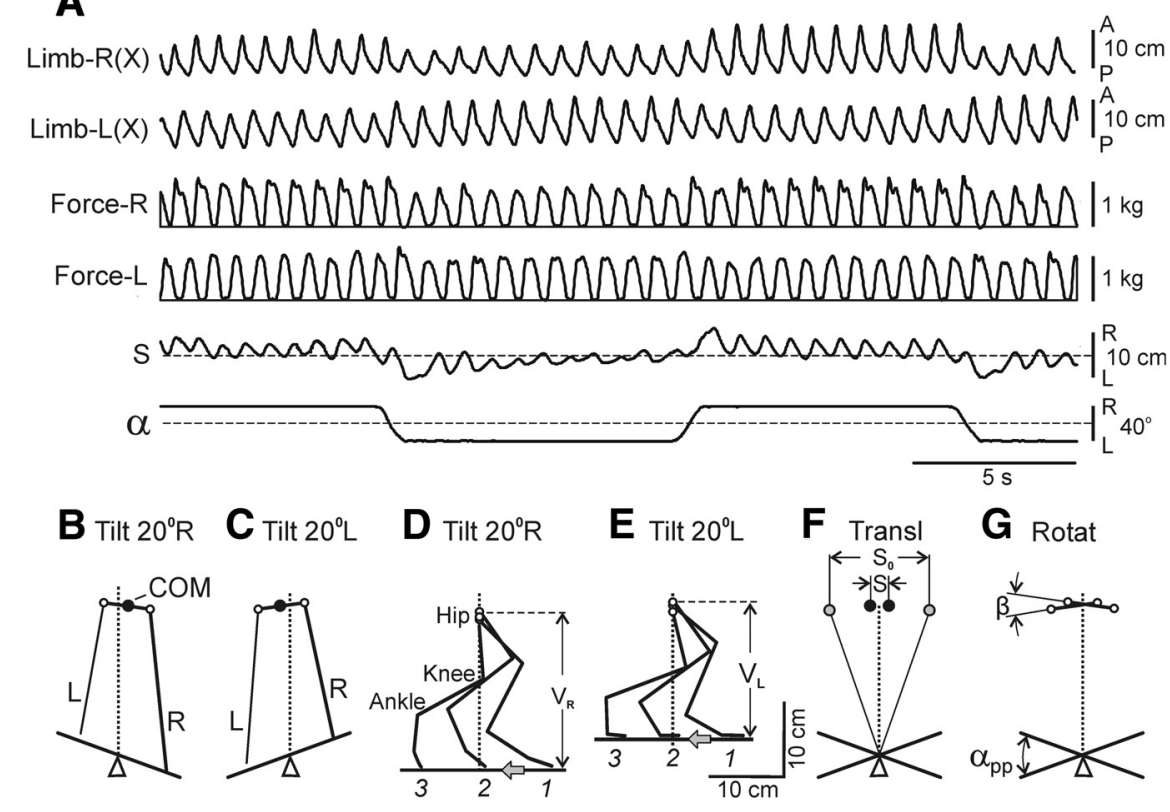

\section{$\mathrm{H}$}

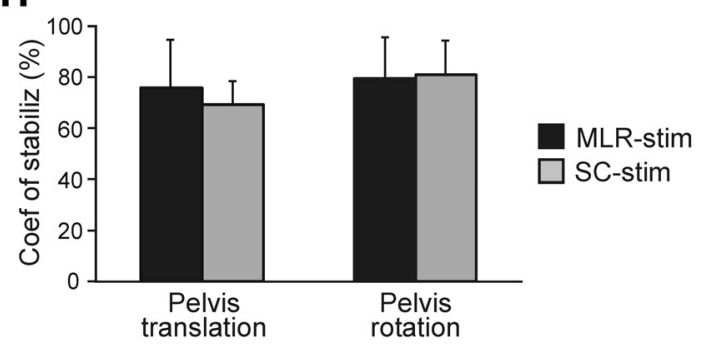

Figure 5. Effects of lateral tilt of treadmill (Test 2). $\boldsymbol{A}$, Movements and forces of the right and left limbs, as well as horizontal pelvis oscillations $(S)$. The treadmill was periodically tilted to the right and to the left $(\alpha)$. $\boldsymbol{B}, \boldsymbol{C}$, Configuration of hindquarters (rear view) on the right-tilted $(\boldsymbol{B})$ and left-tilted $(\boldsymbol{C})$ treadmill. $\boldsymbol{D}, \boldsymbol{E}$, Stick diagram of the right limb at three phases of its stance $(1,2,3)$ when walking on the right-tilted $(\boldsymbol{D})$ and left-tilted $(\boldsymbol{E})$ treadmill. $V_{R}$ and $V_{L}$ is the functional (vertical) length of the limb at the corresponding tilt. The gray arrows show the direction of treadmill motion. $\boldsymbol{F}-\boldsymbol{H}$, Estimation of the efficacy of postural corrections. $\boldsymbol{F}$, Two extreme positions of the anus (black circles; taken from $\boldsymbol{B}, \boldsymbol{C}$ ) and two extreme positions of the marker attached to the treadmill at height of anus (gray circles). $\boldsymbol{G}$, Two extreme angles of the pelvis tilt (taken from $\boldsymbol{B}, \boldsymbol{C}$ ) and two extreme angles of the treadmill tilt. $\boldsymbol{H}$, The coefficient of stabilization for the pelvis translation and pelvis rotation (mean \pm SD), in cats with MLRstimulation ( $N=4, n=16$ and 14 , respectively) and SC-stimulation ( $N=3, n=14$ and 8 , respectively).

$\left(\sim 90^{\circ}\right)$ relative to the pelvis, during both inward and outward steps, as well as during MLR and SC-evoked locomotion (Fig. $3 G)$. We suggest that this angle was stabilized by a transfer mechanism of each limb, which contributes to the normal configuration of the hindquarters in the transverse plane (see Limb transfer mechanism).

\section{Reactions to lateral tilt}

We have found that all decerebrate cats with unrestrained pelvis $(N=6)$ were able to walk and keep balance on the laterally tilted treadmill (Fig. 1C,D; Test 2), as illustrated in Figure 5A. With a trapezoidal profile of tilts, the cat exhibited rapid reactions to inclinations of the support surface to the right and to the left, and well adapted its locomotor pattern to a steady inclination of the walkway.

Figure $5 B, C$, shows the configuration of hindquarters (rear view) at the mid-stance of the right limb (and the transfer of the left limb) when the cat was walking on the tilted treadmill. With the right treadmill tilt (Fig. 5B), the pelvis was displaced horizontally to the right (in relation to the sagittal plane of the fixed
G Rotat

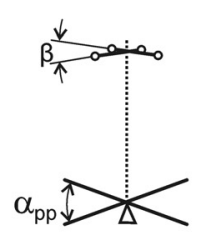

forequarters) and turned clockwise. With the left treadmill tilt (Fig. 5C), it was displaced to the left and turned counterclockwise. Figure 5D, E, shows the configuration of the right limb (side view) during the right (Fig. 5D) and left (Fig. $5 E$ ) tilt of the treadmill, observed at the onset (1), middle (2), and end (3) of the stance phase. These changes in the limb configuration caused changes in its "vertical length" $\left(V_{R}\right.$ and $\left.V_{L}\right)$. The changes of vertical length of the left and right limb during tilt reduced both the pelvis horizontal translation and rotation.

To estimate the efficacy of postural corrections when the cat was walking on the inclined support surface, we used two approaches. First, we determined the mean COM position in each step cycle. This was done for 10 sequential cycles with the treadmill tilted to the right and for 10 cycles with its tilt to the left. Then the mean COM positions were averaged across each sequence. The difference between the two values (Fig. 5F; S) was compared with the corresponding difference expected in the absence of postural corrections $\left(S_{0}\right)$. To estimate $S_{0}$, a reference marker was rigidly attached to the treadmill above the axis of tilts and at the height of anus, and excursions of this marker were measured (Fig. 5F; Beloozerova et al., 2003). Second, we averaged the mean angles of pelvis orientation in 10 steps when the treadmill was tilted to the right and in 10 steps when it was tilted to the left. The difference between the two values $(\beta)$ was compared with the difference expected in the absence of postural corrections, i.e., to the change in the treadmill angle $\left(\alpha_{\mathrm{PP}}=40^{\circ}\right.$; Fig. $\left.5 G\right)$. The coefficient of stabilization was then calculated for the translational and rotational pelvis motions:

$$
\mathrm{K}_{\mathrm{TRAN}}=1-\mathrm{S} / \mathrm{S}_{0} ; \mathrm{K}_{\mathrm{ROT}}=1-\beta / \alpha_{\mathrm{PP}} .
$$

As shown in Figure $5 H$, the coefficient of stabilization was $\sim 80 \%$ for both pelvis motions, as well as during locomotion evoked by MLR stimulation and by SC stimulation. These results demonstrated the high efficacy of the system maintaining the dorsalside-up body orientation in the decerebrate cat walking on the inclined surface.

\section{Neural mechanisms of postural corrections}

Limb transfer mechanism

To test the hypothesis that there is a mechanism for transferring the limb to a standard (in relation to the pelvis) initial stance position (see above), the cats $(N=6)$ were subjected to Tests 3 and 4 (Fig. $1 F)$.

Figure $6 A$ illustrates the effect of leftward translation of the supporting surface under the left limb (Test 3) during SC-evoked locomotion. A trajectory of the left foot in X/Y coordinates (see 
Materials and Methods) during two steps is shown. In the stance phase of the step cycle preceding the perturbation (thick black line, points 1-10), the left foot was moving backward (along the trajectory $Y=2 \mathrm{~cm}$ ) at the speed of the treadmill motion. During the following transfer phase (thin black line), the foot moved forward to land at the position (point 14) very close to the initial stance position (point 1). Then, the stance phase of a new cycle began (thick red line), with the foot trajectory very close to that in the previous cycle. However, at point 18, a fast leftward translation of the supporting surface was performed, which resulted in shifting the foot to a new, more lateral stance position (point 19; $Y=11 \mathrm{~cm}$ ). From point 19, the stance continued at this new lateral position until point 23, where a new transfer phase began (thin red line). During this phase (points 24-27), the foot was moved to the position (point 27; $Y=3 \mathrm{~cm}$ ), which was very close to the initial positions in the two preceding steps (points 1 and 14, respectively). The time required for correcting the step perturbation (the difference between the time points 18 and 27) was $\sim 0.4 \mathrm{~s}$.

This type of corrections, i.e., a displacement of the affected limb from a perturbed lateral position to a standard unperturbed lateral position, performed during the transfer phase of the step cycle, was observed in all tested cats, during both outward support translation (Fig. $6 B, C$ ) and inward translation (Fig. 6D,E). The obtained results were similar for SC-stimulated cats (Fig. $6 B, D)$ and for MLR-stimulated cats (Fig. 6C,E).

In these experiments, we also recorded the lateral $(Y)$ position of the opposite (unaffected) limb in the cycles before, during, and after the perturbation (Fig. $6 B-E$ ). One can see that changes in the stance position of this limb were much smaller than changes in the affected limb (though in Fig. $6 B, C, E$, the changes were statistically significant). This finding indicates that, with the pelvis fixed, a reaction to support translation is confined mainly to the affected limb.

To analyze the mechanism for transferring the limb to a standard stance position, we applied a lateral force to the limb (by means of an elastic string), which did not evoke displacement of the limb during the stance phase, but affected the limb during the whole swing phase (Test 4; Fig. $1 F$ ). Figure $7 A$ illustrates changes in the step trajectory caused by application of the lateral force. The left foot trajectory in the unperturbed step cycle is shown, with its transfer phase (points 1-5), and stance phase (points 5-12) indicated by thick and thin black lines, respectively. The stance trajectory had a lateral coordinate $Y=2 \mathrm{~cm}$. Then a lateral force (in the range of $0.15-0.2 \mathrm{~kg}$ ) was applied to the area around the ankle joint (point 13), which resulted in an increasing lateral deviation of the limb during the transfer phase (thin red line) that reached a maximum $(Y=13 \mathrm{~cm})$ at point 17 . After this point, the limb deviation was gradually decreasing, and the foot finally landed at $Y=4 \mathrm{~cm}$ (point 19). A new stance trajectory (thick red line) was slightly displaced to the left as compared with the previous cycle (from $Y=2.5 \mathrm{~cm}$ to $Y=4 \mathrm{~cm}$ ). One can conclude that during transfer phase the limb developed an active lateral force to
Translation outward

B
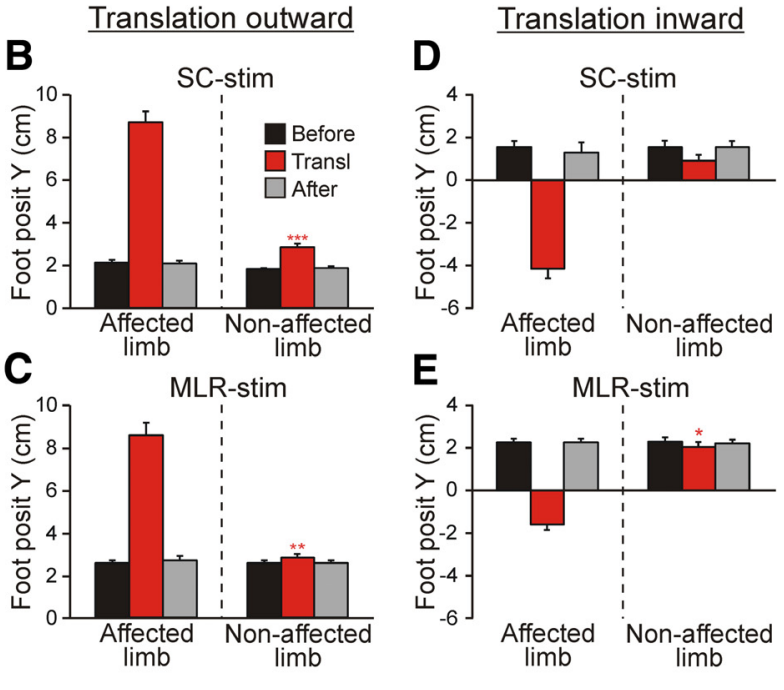

Figure 6. Effect of support translation during stance (Test 3). $\boldsymbol{A}$, Leftward translation of the supporting surface under the left

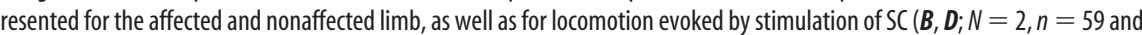
$N=2, n=14$, respectively) and $\operatorname{MLR}\left(\boldsymbol{C}, \boldsymbol{E} ; N=2, n=40\right.$ and $N=2, n=60$, respectively). Indication of significance level: ${ }^{*} p<$

overcome the applied external force, which resulted in returning the limb to the position close to the standard one.

Figure $7 B, C$, shows effects of the lateral pulling performed continuously during many sequential steps in cats with SC and MLR-evoked locomotion, respectively. The applied force $(0.15-$ $0.2 \mathrm{~kg}$ ) did not affect the limb position during stance. During swing, this force caused a large lateral deviation of the limb in each step ( similar to that shown in Fig. 7A), with its peak $Y=$ 12-14 cm. However, due to the counteracting force, the limb landed at a much more medial position (with $Y=4-6 \mathrm{~cm}$ ), giving rise to a stance trajectory with this $Y$ value. Such results, demonstrating stabilization of the landing limb position, were obtained in all tested cats, both with SC-evoked locomotion (Fig. 7D) and with MLR-evoked locomotion (Fig. 7E). Similar result was obtained with application of the constant force in the opposite (medial) direction (not illustrated).

One could expect that, to overcome the limb abduction or adduction caused by pulling, the muscles with an opposite function (limb adduction or abduction) will be activated, and their antagonists (abductors or adductors) will be inactivated. We have not found, however, any significant changes in the activity during the transfer phase (in steps with lateral or medial limb pulling) in 10 recorded hindlimb muscles (see Materials and Methods). Small changes were seen in EMGs of adductor femoris (hip extensor and adductor) and gluteus (hip extensor and abductor) in the steps with and without lateral pulling. Figure $7 F-I$, shows average EMGs of Add-L and Glut-L in different phases of the step cycle. One can see that both muscles exhibited a high activity in the stance phase (which corresponds to their extensor function), whereas their activity in the transfer phase was relatively low (Fig. 7F,H). Only with larger amplification of EMG signals (Fig. 7G,I) one can see some increase of Add-L activity in the transfer phase during pulling, as well as a small decrease of Glut-L activity in this phase.

One possible explanation for the relatively low activity of adductor and abductor muscles in the swing phase is that a support of 

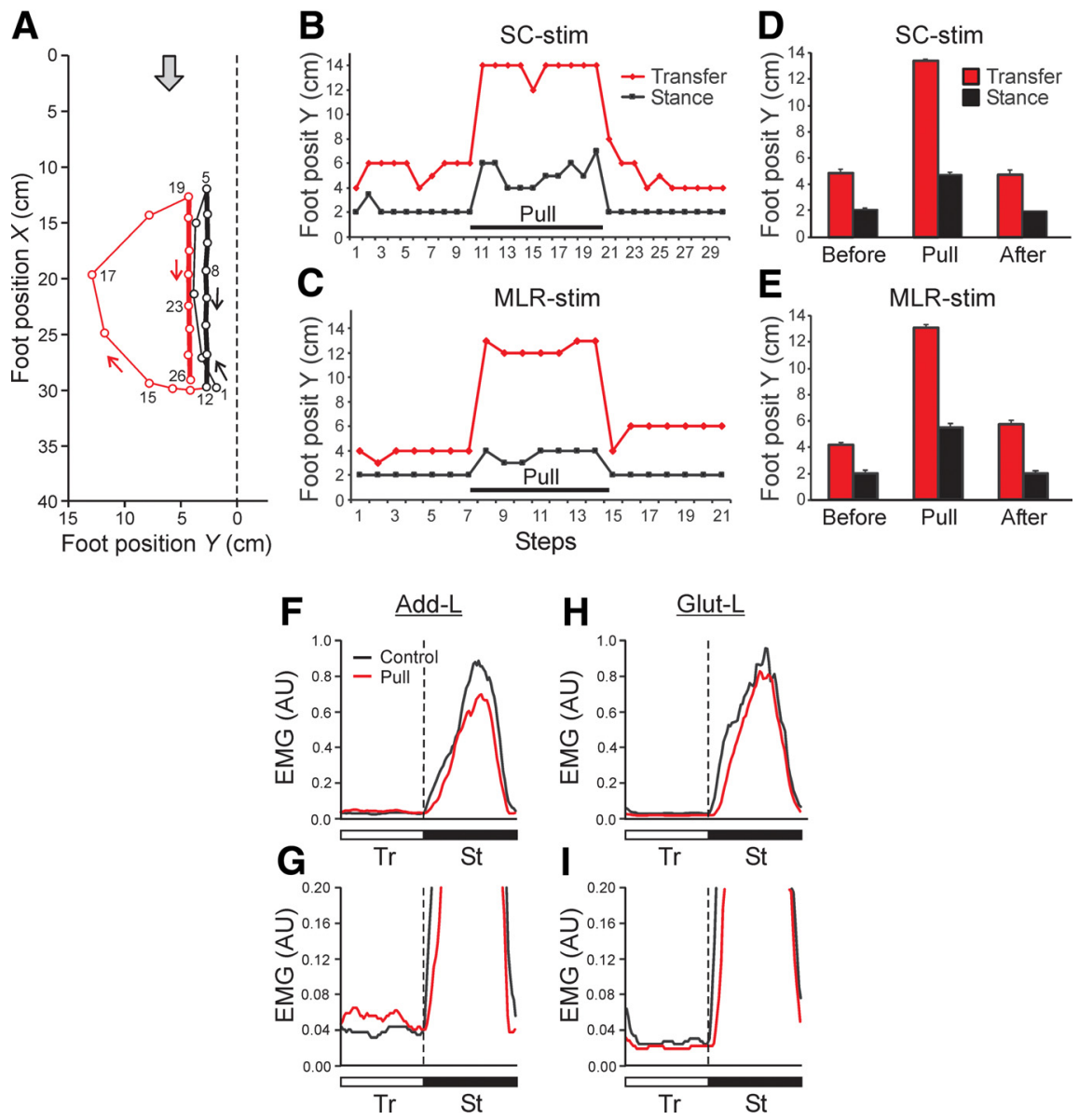

Figure 7. Effect of lateral limb pulling (Test 4).A, Leftward pulling of the left limb during MLR-evoked locomotion. Sequential positions ( $40 \mathrm{~ms}$ between frames) of the left foot during two step cycles are shown. The foot trajectories in two cycles are shown by thick lines in stance, and by thin lines in swing; black and red lines indicate the unperturbed and perturbed steps, respectively. The gray arrow shows the direction of treadmill motion. $\boldsymbol{B}, \boldsymbol{C}$, The effect of limb pulling that was applied continuously during 10 sequential steps (Pull). The lateral limb position in stance (black line) and its peak deviation in swing (red line) are shown. $\boldsymbol{D}, \boldsymbol{E}$, The lateral foot position (mean $\pm \mathrm{SE}$ ) during swing and stance in the steps before pulling (Before), during pulling (Pull), and after pulling (After). Data are presented for locomotion evoked by stimulation of the $\operatorname{SC}(\boldsymbol{B}, \boldsymbol{D})$ and $\operatorname{MLR}(\boldsymbol{C}, \boldsymbol{E})$. $\boldsymbol{D}, \boldsymbol{E}$, Before, Pull, After: $n=15,36,15$, and $n=25,28,7$, respectively. $\boldsymbol{F}-\boldsymbol{I}$, Effects of lateral pulling on EMGs of Add-L $(\boldsymbol{F}, \boldsymbol{G})$ and $G$ lut-L $(\boldsymbol{H}, \boldsymbol{I})$; the EMGs were rectified and averaged over 10 steps. The EMGs (arbitrary units, $A U$ ) are presented with low amplification $(\boldsymbol{F}, \boldsymbol{H})$ and with high amplification $(\mathbf{G}, \boldsymbol{I})$. The EMG for unperturbed steps (Control) is superimposed on the EMG for perturbed steps (with lateral pulling, Pull). Tr and St phases of the step cycle are indicated.

the body weight during stance needs a much higher activity of these bifunctional muscles than is needed for correcting the limb trajectory during swing. We cannot exclude, however, that some nonrecorded muscles (from $\sim 20$ muscles acting around the hip joint of the cat; Reighard and Jennings, 1902) would be more specifically involved in the active control of lateral limb position during swing.

\section{Limb displacement compensation mechanism}

Under unrestrained pelvis condition (Type 1 experiments; Fig. 1B), a lateral push applied in the stance phase of a limb caused activation of abductor or adductor muscles of this limb, depending on the push direction (Fig. $8 A, B$ ). A push in the stance phase of the contralateral limb (Push in; Fig. $8 A$ ) caused an increase in the activity of the abductor (Glut-R). A push in the stance phase of the ipsilateral limb (Push out; Fig. $8 B$ ) caused an increase in the activity of the adductor (Grac-L). Similar reactions were found in all tested cats, with locomotion evoked by MLR-stimulation $(N=2, n=27)$ or by SC-stimulation $(N=2, n=24)$. These reactions were similar to those observed in intact cats (Karayannidou et al., 2009a), suggesting that the basic neuronal networks underlying these reactions are located at the brainstem-cerebellum-spinal level.

A number of afferent inputs can contribute to these reactions. In particular, lateral displacements of the pelvis (Fig. $8 A, B ; \mathrm{S})$ in the stance phase can cause abduction or adduction of the limb relative to the trunk, which can be monitored by stretch receptors of the corresponding muscles. To reveal the role of these signals, the pelvis was rigidly fixed, and the stepping limb was translated (in the stance phase) inward or outward by moving the supporting plate positioned on the treadmill belt (Test 4; Fig. $1 F$ ). The results of these experiments are illustrated in Figure $8 C, D$. Inward translation (Fig. 8C; Transl in) caused limb adduction and activation of Glut (hip abductor). Outward translation (Fig. 8D; Transl out) caused limb abduction and activation of Grac (hip adductor), as well as inactivation of Glut. Similar reactions were observed in all tested cats with locomotion evoked by MLR-stimulation $(N=2, n=36)$ and by SC-stimulation $(N=4, n=39)$. Thus, signals about limb abduction and adduction during the stance phase contribute to the activation of the limb stance mechanism. One can suggest that this mechanism counteracts the lateral displacements and tilts of the hindquarters caused by lateral pushes.

\section{Limb load compensation mechanism}

For studying the effect of loading/unloading of the stepping limb, the pelvis was rigidly fixed, and the treadmill was tilted to the right or to the left (Test 5; Fig. $1 G, H)$. These tilts caused upward or downward translation of the supporting surface (small changes in the surface inclination under each limb were not taken into account) causing simultaneous loading of one limb and unloading of the other one. A representative example of effects of limb loading/unloading on EMGs and force is shown in Figure 9A. To evaluate these reactions, the force and EMGs were averaged for the ipsilateral (blue, $n=12$ ) and contralateral (red, $n=12$ ) tilts (Fig. 9B). The amplitude of force and EMGs in a given limb were larger when the support surface was translated upward (contralateral tilt, limb loading) compared with its translation downward (ipsilateral tilt, limb unloading). An increase of EMGs in the loaded limb was observed not only in the extensors (Vast, Gast) but also in the flexor (Tib), thus contributing to an increase of the limb stiffness. Similar reactions were found in all tested cats $(N=3, n=9)$. In the unrestrained cat, these limb load compensation mechanism would counteract the displacement of hindquarters in the direction of the surface tilt (see Discussion).

\section{Trunk configuration mechanism}

To reveal the role of the trunk mechanism for balance control, we recorded bilaterally the EMGs of back muscles (erector spinae and multifidus). The activity of these two muscles during loco- 
A
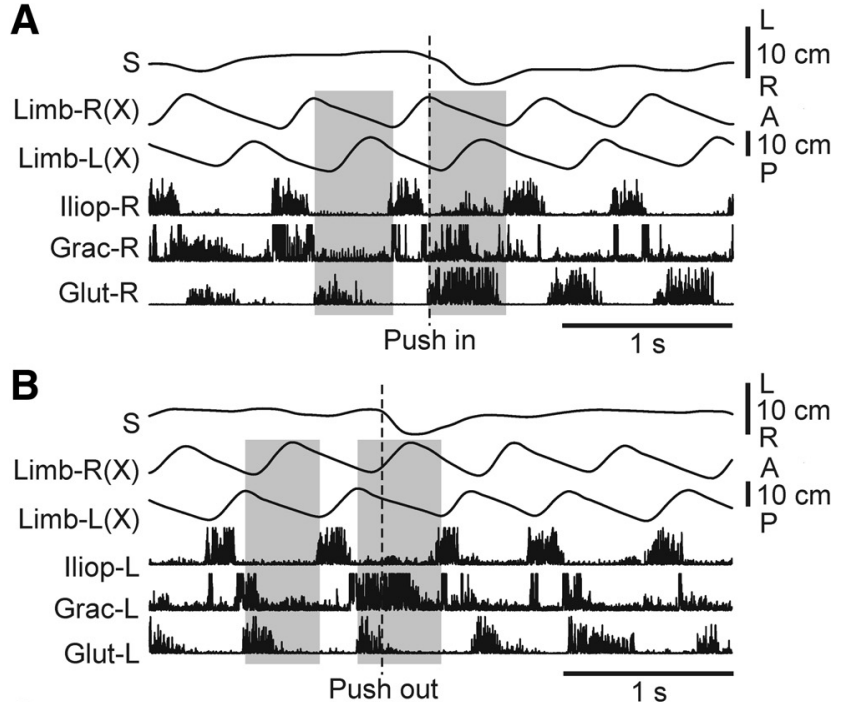

C

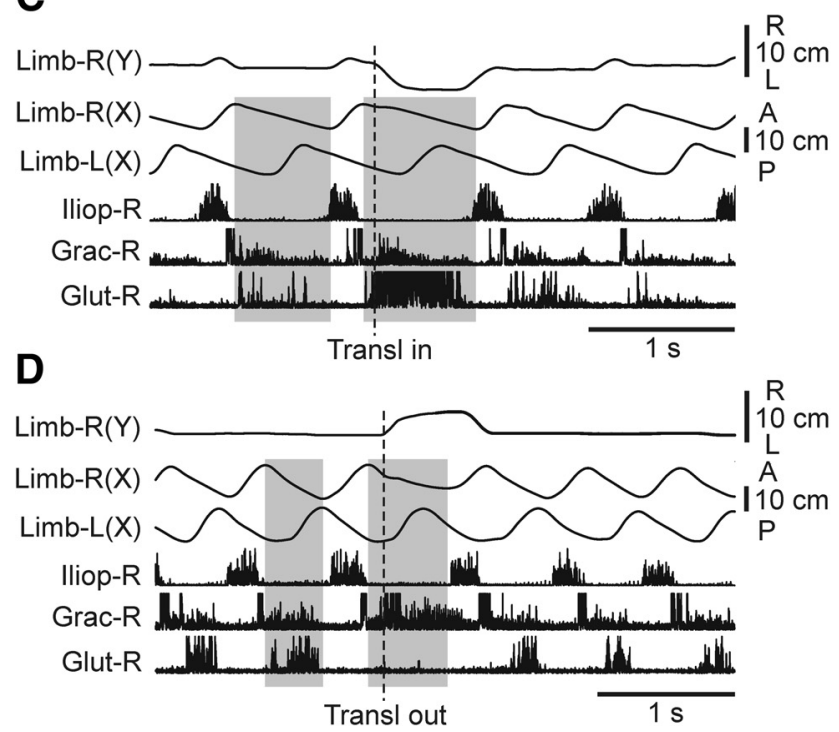

Figure 8. Effects of push and support translation on EMGs. $A-D$, Representative examples of EMG responses to push and support translation in 3 selected muscles of the limb performing the stance at the moment of disturbance. $A, B$, Effects of rightward push during stance of the right $\operatorname{limb}(\boldsymbol{A}$, Push in) and during stance of the left limb (B, Push out; Test 1$) . \boldsymbol{C}, \boldsymbol{D}$, Effects of inward (C, Trans in) and outward ( $\boldsymbol{D}$, Trans out) limb translation during stance (Test 3 ). Shaded area indicates the stance phase of the right limb (Limb-R) in $\boldsymbol{A}, \boldsymbol{C}, \boldsymbol{D}$ and of the left limb (Limb-L) in $\boldsymbol{B}$, in the step preceding disturbance and in the step with disturbance. Limb (Y): mediolateral limb position. Other designations as in Figure 5 .

A Limb-R(x)WWWMWMW15

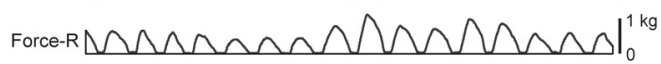

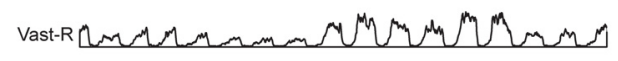

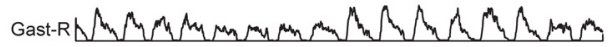

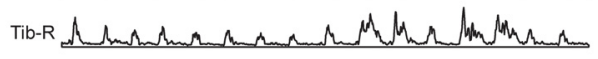

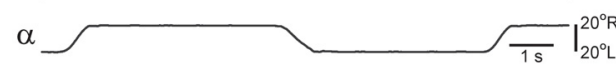

B

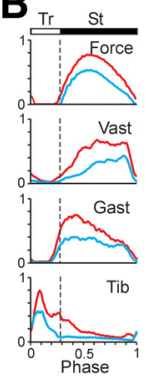

Figure 9. Effects of limb loading (Test 5). $\boldsymbol{A}$, In the cat walking on the tilting treadmill (pelvis fixed), stepping movements of the right limb, its contact force, and EMGs were recorded. $\boldsymbol{B}$, The averaged force and EMGs ( $n=12$ ) for the ipsilateral (blue) and contralateral (red) tilts.

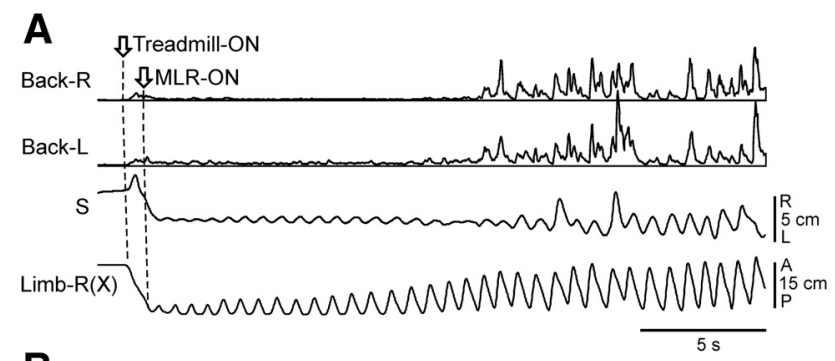

B

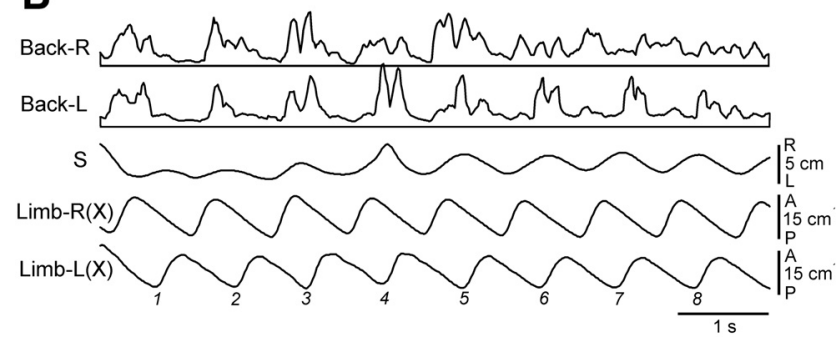

Figure 10. Activity of back muscles during locomotion (Type 1 experiment). $A$, Parallel activation of back muscles (left and right erector spinae, Back-L and Back-R) and of mechanisms of stepping caused by MLR-stimulation in the cat with unrestrained pelvis. B, Step-related modulation of back muscles (erector spinae) during MLR-evoked locomotion. Abbreviations and designations as in Figure 5.

motion as well as their reactions to postural disturbances were similar. These muscles in decerebrate cats were not active at rest. The activity appeared with MLR or SC stimulation, along with the development of stepping movements, as illustrated in Figure $10 \mathrm{~A}$. One can see that MLR stimulation caused a gradual bilateral activation of back muscles and enhancement of stepping movements (an increase in the step amplitude and appearance of the lateral body oscillations). Activation of these two systems resulted in lifting the hindquarters and maintaining their dorsal side up orientation during walking. Step-related modulation was often observed in the activity of back muscles, as illustrated in Figure $10 B$. The right and the left back muscles could be modulated in-phase (cycles 1-5), or modulated on only one side (cycles 6-8), or modulated in anti-phase (not illustrated). Modulation of back muscles in walking intact and decerebrate cats was described by Carlson et al. (1979) and Zomlefer et al. (1984), respectively. This modulation is partly due to the influences from the locomotor central pattern generator (Koehler et al., 1984) and its primary role is to control the stiffness of the back (Carlson et al., 1979).

We studied responses of back muscles to different postural perturbations. In Test 1 (Fig. 1B), a brief pulse of the lateral force (push) was applied to the pelvis. The push caused a rapid displacement of the pelvis, trunk bending (Fig. $3 \mathrm{C}$ ), and adduction or abduction of the supporting limb. A representative example of back muscles response to push is shown in Figure 11A. One can see that push in any direction caused coactivation of left and right back muscles. Similar reactions were observed in all tested cats with both MLR- and SC-stimulation $(N=2, n=12$ and $N=3$, $n=18$, respectively).

To determine afferent sources of these responses (limb or trunk afferents), two tests were performed. In Test 3 (pelvis restrained; Fig. $1 F$ ), we moved a limb relative to trunk by lateral translating the support surface under it. The translation for $\sim 7$ $\mathrm{cm}$ evoked some bilateral response in back muscles (Fig. 11B), suggesting that limb afferents contribute to back muscles activation. Then the same cat was subjected to Test 6 (Fig. $1 J$ ). The 

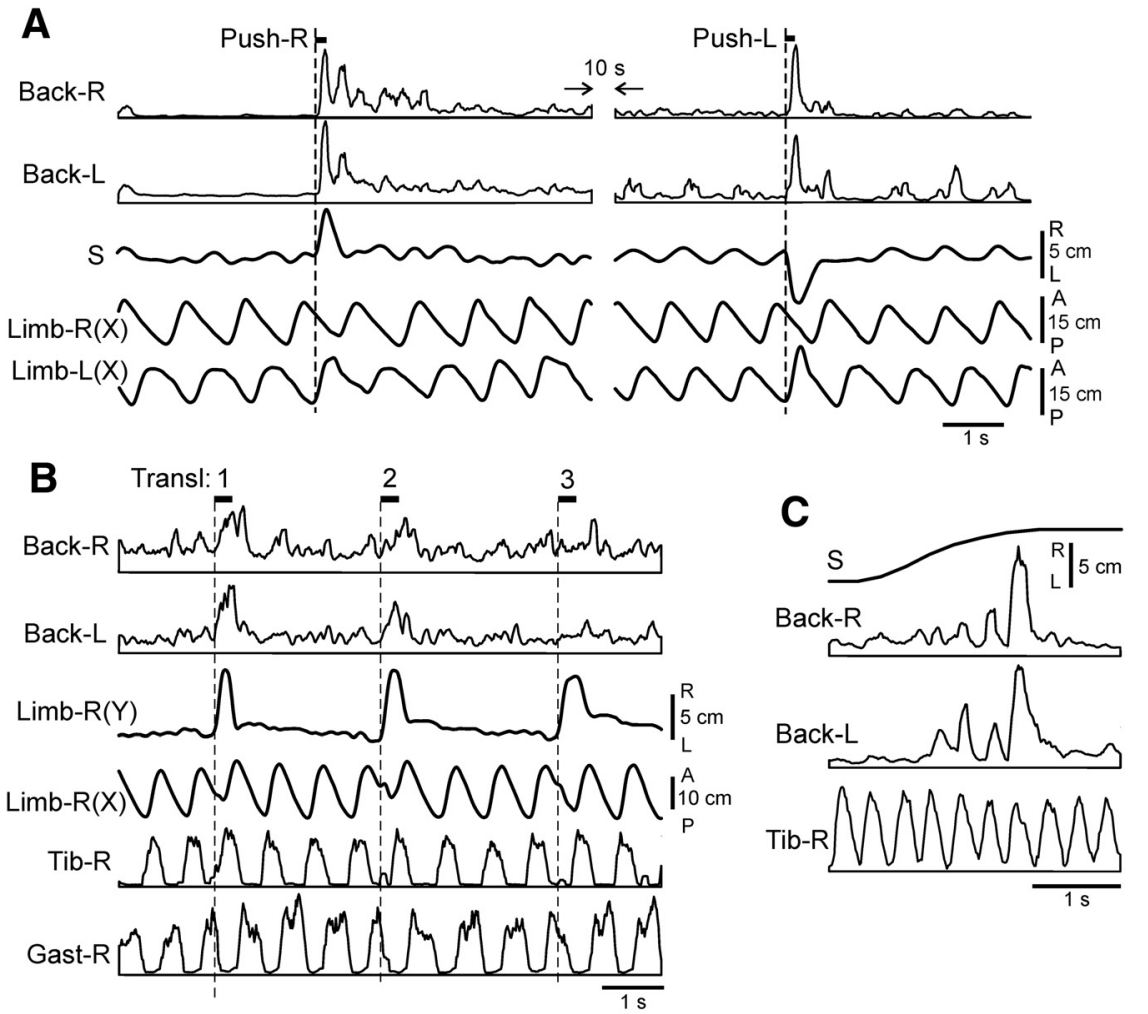

\section{D}

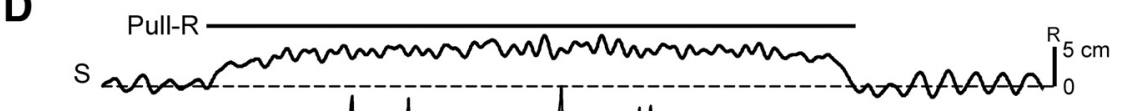

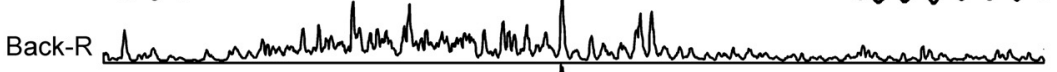

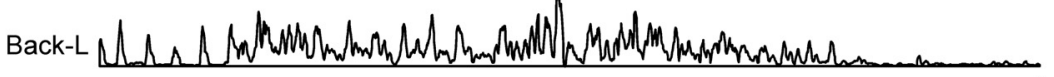
Limb-R(x) WWWWWWWWWWWWWW

Figure 11. Responses of back muscles to different disturbances during locomotion. $A$, Responses of the left and right erector spinae to the rightward and leftward pushes applied during MLR-evoked locomotion in the cat with unrestrained pelvis (Test 1). $\boldsymbol{B}$, Responses of the right and left multifidus to a lateral translation of the right limb support during MLR-evoked locomotion in the cat with restrained pelvis (Test 3). $C, D$, Responses of the right and left multifidus to a lateral translation of the pelvis in the suspended cat performing air stepping caused by MLR-stimulation $(\boldsymbol{C})$ and SC-stimulation (D; Test 6 ). In $\boldsymbol{D}$, the long-lasting lateral trunk bending (by pulling the pelvis to the right, Pull-R) was performed. Amplification for Back-L and Back-R in $\boldsymbol{B}$ was two times smaller than in C. Abbreviations and designations as in Figures 5 and 8.

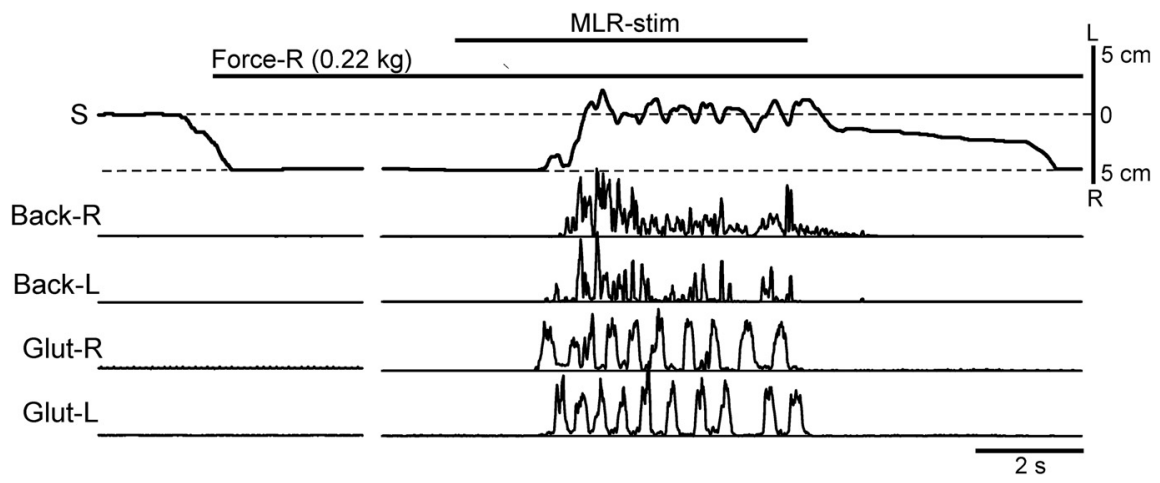

Figure 12. Reflex responses to trunk bending at rest and during locomotion (Test 7). At rest, a lateral force applied to the pelvis (Force-R) in the suspended cat caused trunk bending. Stimulation of MLR caused air-stepping (monitored by Glut-R and Glut-L EMGs) and bilateral activation of back muscles (left and right erector spinae), which resulted in straightening of the trunk despite continuous application of the lateral force. Abbreviations and designations as in Figures 5 and 8. treadmill was removed and air stepping was evoked in the suspended hindquarters. As shown in Figure 11C, displacement of the pelvis together with the hindlimbs (by moving the pelvis restraint by $6 \mathrm{~cm}$ ) caused a large bilateral activation of back muscles, whereas rhythmical limb stepping movements (monitored by Tib-R recording) were not affected. Similar results in Tests 3 and 6 were obtained in all $(N=3)$ tested cats ( $n=29$ and $n=12$, respectively).

The results of Tests 6 and 3 suggest that flexion of the trunk caused by push (Fig. $11 A$ ) was the main factor eliciting responses in back muscles, whereas distortion of stepping limb movements had much smaller contribution (Fig. 11B,C, compare back muscles responses).

Back muscles responded to sensory input not only dynamically (Fig. $11 A, C$ ) but also statically. Figure $11 D$ shows that a long-lasting lateral displacement of the pelvis (caused by lateral pull; Fig. $1 K$, Test 7) resulted in the long-lasting trunk bending and coactivation of Back- $\mathrm{R}$ and Back-L. The likely sensory input for this reaction was that signaling an extent of bending.

We suggested that coactivation of back muscles (caused by trunk bending) leads to an increase in the trunk stiffness (Hu et al., 2009), and therefore to a reduction in the externally caused trunk bending, which will result in a postural correction. This hypothesis was tested in the experiments with suspended hindquarters (Fig. $1 K, L$, Test 7$)$. As shown in Figure 12, with no MLR stimulation, a lateral force $(0.22$ $\mathrm{kg}$ ) caused a rightward flexion of the trunk and displacement of the pelvis by $\sim 5 \mathrm{~cm}$. MLR stimulation activated the locomotor mechanisms and evoked air stepping (monitored by Glut-R and Glut-L recording). It also caused a bilateral activation of the back muscles, which resulted in trunk straightening (despite the continuous action of the lateral force). These effects were similar when locomotion was evoked by MLR stimulation $(N=3, n=6)$ or by SC stimulation $(N=$ $1, n=4)$. This result strongly supports our hypothesis that the trunk-stabilizing mechanism is based on the feedback control of the trunk stiffness.

\section{Discussion}

In the first part of the present study, we examined the capacity of the decerebrate cat to compensate for two destabilizing factors applied during locomotion: a brief lateral push and a sustained lateral tilt of the treadmill. We show that the cat was 
able to rapidly restore balance after push, using two types of postural reactions, the outward and inward steps (Fig. 3). The type of reaction depended on the push direction and on the phase of push application in the step cycle. The outward step (with the limb landing more laterally than during normal steps) occurred when the push was applied during swing of the contralateral limb. The inward step (with the limb landing more medially than during normal steps) was performed when the push was applied during swing of the ipsilateral limb. These two types of reactions to lateral push were observed also in the walking intact cat (Karayannidou et al., 2009a) suggesting that, in both cases, the reactions were generated by the same neuronal circuits residing at the brainstemcerebellar-spinal level. The forebrain mechanisms (motor cortex in particular) seem to play a secondary role by scaling these reactions (Hof, 1996). In walking humans, lateral balance can be corrected by foot placement (Townsend, 1985; Kuo, 1999), and a lateral push also evokes outward or inward step, depending on the phase of perturbation (Hof et al., 2010, Hof and Duysens, 2013).

Our results also show that the decerebrate cat was able to walk on the laterally tilted treadmill. This postural adaptation was based, to a large extent, on the transformation of the normal (symmetrical) locomotor pattern into the asymmetrical one, with different functional lengths of the right and left limbs (Fig. 5D,E). A similar adaptation to an inclined walkway was observed in walking intact cats (Karayannidou et al., 2009b) suggesting that, in both cases, the basic pattern of reactions was due to the activity of the same brainstemcerebellar-spinal mechanisms. Despite the fact that in intact cats the activity of the motor cortex correlates with the inclination of the walkway (Karayannidou et al., 2009b), one can conclude that these cortical commands (and signals from other forebrain centers) may play only a secondary role in this postural adaptation.

In a recent study it was shown that the walking decerebrate cat was able to keep balance when a lateral pull was applied to its tail (Musienko et al., 2012a). Together with the results of the present study, these findings show that neuronal mechanisms of the brainstem, cerebellum, and spinal cord are able to maintain lateral stability during locomotion and to compensate for a wide range of destabilizing factors. Decerebrate animals are also able to generate appropriate postural reactions to different perturbations during standing (Musienko et al., 2008; Honeycutt et al., 2009; Honeycutt and Nichols, 2010). One can thus conclude that the feedback control of posture and bal-

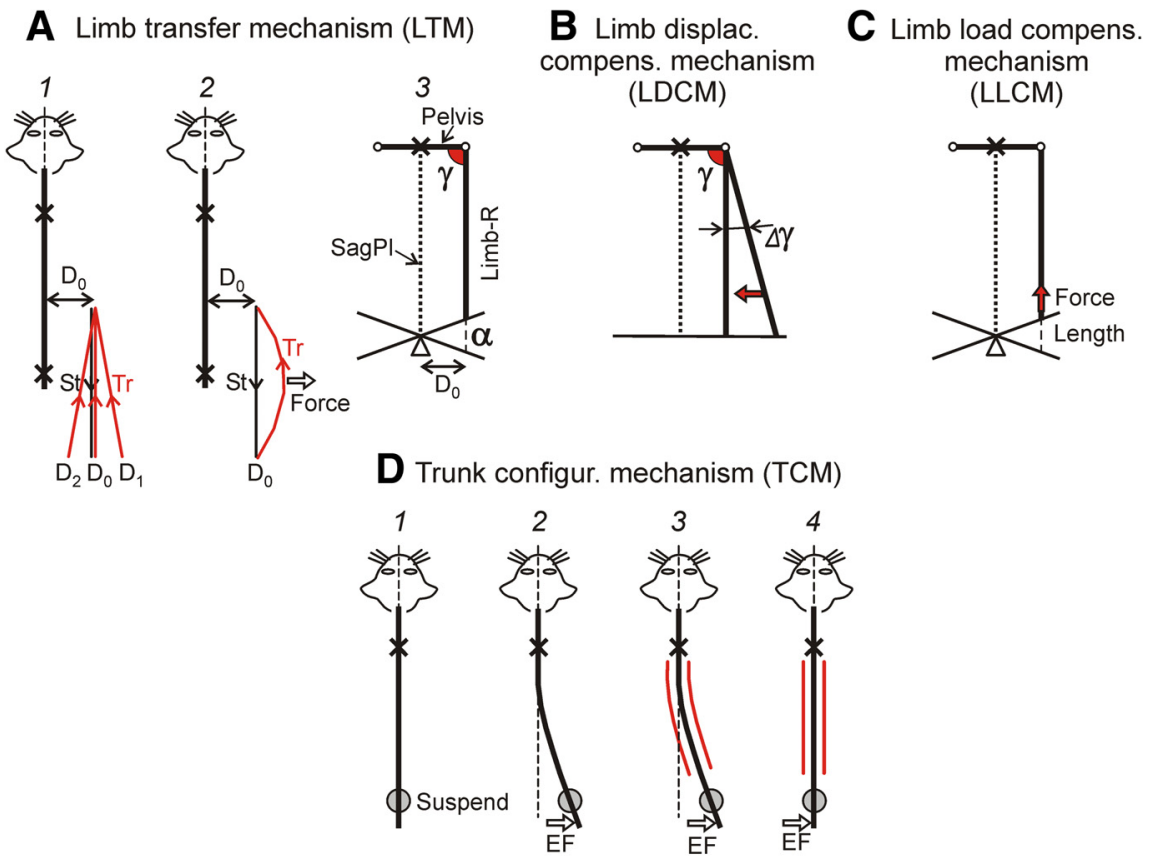

E Integration of limb and trunk mechanisms
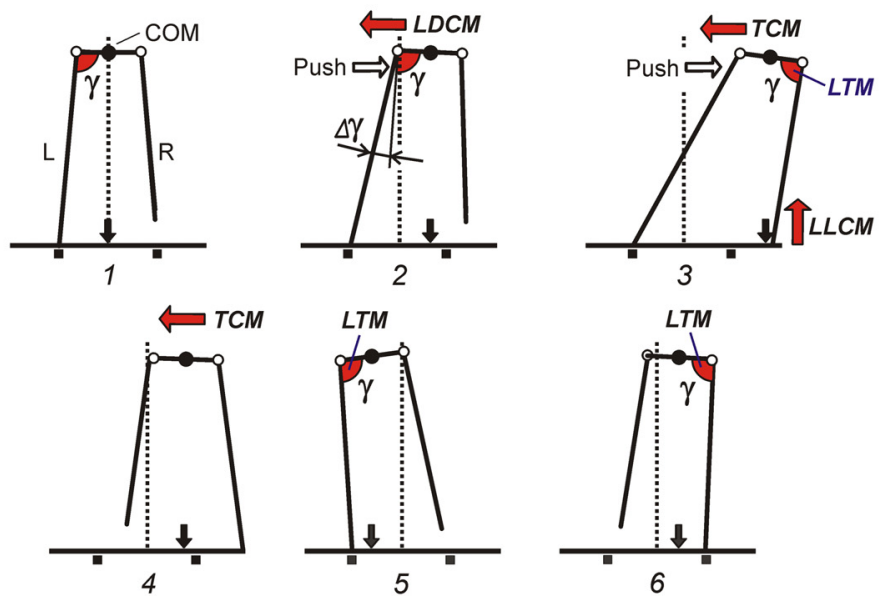

Figure 13. Four basic mechanisms for balance control during locomotion. $\boldsymbol{A}$, The limb transfer mechanism controls the limb landing position. $\boldsymbol{B}$, The limb displacement compensation mechanism regulates the limb abduction-adduction during stance. $\boldsymbol{C}$, The limb load compensation mechanism controls the extensor activity during stance. $\boldsymbol{D}$, The trunk configuration mechanism controls the trunk shape. Crosses and circles indicate the points of body fixation and suspension, respectively, which allow isolation of the different mechanisms. See text for explanations. $\boldsymbol{E}$, Integration of limb and trunk postural mechanisms in the reaction to lateral push. E1, Unperturbed configuration of the hindquarters during locomotion. E2, Push to the right causes a rightward displacement of the pelvis accompanied by abduction $(\Delta \gamma)$ of the supporting (left) limb in relation to the trunk. This abduction triggers $L D C M$, which counteracts the lateral pelvis displacement and reduces its value. $\boldsymbol{E 3}$, At the same time the right limb, due to the activity of its LTM, is transferred forward and landed at a standard angle $\gamma$ in relation to the pelvis. However, because of the trunk bending, it lands at a much more lateral position than the stance position in normal steps (indicated by small squares). After landing of the right limb, its LLCM comes into operation. Also, the push-caused lateral trunk bending activates TCM, which results in the trunk straightening $(\boldsymbol{E} \mathbf{3}, \boldsymbol{E} 4)$ during the St phase of the right limb. Because of the restored straight trunk configuration, LTM causes landing of the left and right limbs in the subsequent steps (E5 and $\boldsymbol{E} 6$ ) at the normal mediolateral position. Thus, due to the operation of the limb and trunk mechanisms, the normal locomotor body configuration is restored.

ance under different conditions is basically the hindbrain and spinal cord function.

In the second part of the present study, we analyzed the four basic mechanisms participating in the postural reactions to pushes and tilts in walking animals. These mechanisms are schematically shown in Figure 13, A-D. 
The limb transfer mechanism (LTM) revealed in the experiments illustrated in Figures 6 and 7 is schematically shown in Figure 13A. Independently on the lateral foot position (relative to trunk) at the onset of swing phase (Fig. 13A1; $\mathrm{D}_{0}, \mathrm{D}_{1}, \mathrm{D}_{2}$, etc.), the final foot position at the moment of limb landing is always the same, $\mathrm{D}_{0}$. Thus, the transfer trajectories, starting at different points, terminate at a standard point and give rise to a standard stance trajectory. The transfer trajectory can be corrected due to the sensory feedback from the "own" limb signaling the current value of hip abduction or adduction (Fig. 13A2), as demonstrated by applying a lateral force to the transferring limb (Fig. 7).

We suggest that the controlled variable in LTM is the angle in the frontal plane between the limb and the trunk (Fig. 13A3; $\gamma$ ). By stabilizing this angle at the end of the transfer phase (usually $\gamma$ is $\sim 90^{\circ}$; Fig. $3 G$ ), the LTM secures a constant laterality of the stance trajectory $\left(\mathrm{D}_{0}\right)$. This relates not only to walking on the horizontal surface but also to walking at different inclinations $(\alpha)$ of this surface (Fig. 13A3).

After the limb has been transferred forward, the limb displacement compensation mechanism (LDCM), based on the reflex responses to changes of the hip angle in the transverse plane (Fig. 8), comes into operation (Fig. 13B). Due to LDCM, an increase of $\gamma$ (Fig. 13B; $\Delta \gamma$ ) or its decrease causes activation of adductors or abductors, respectively. The activated muscles tend to restore the initial hip angle (arrow). The LDCM is driven by sensory feedback from the own limb signaling the angle $\gamma$. Most likely, the afferents providing this input are the stretch receptors of adductor and abductor muscles.

We found that abduction or adduction of the limb during stance caused the landing of the contralateral limb to be in a more abducted or adducted position in relation to the control step, respectively. These crossed effects are very small (Fig. $6 B-E$ ), but one cannot exclude that they contribute to the reaction evoked by a disturbance of balance during locomotion, e.g., to inward and outward steps caused by push. Since the crossed effects were very small, they were not reflected in the angle $\gamma$ (Fig. $3 G$ ).

The limb load compensation mechanism (LLCM) also operates in the stance phase (Fig. 13C). It activates the extensor muscles with loading and flexing the limb (Fig. 9; Orlovsky, 1972; Pratt, 1995; Duysens et al., 2000). The most likely afferents providing input to LLCM are the stretch receptors and Golgi tendon organs of extensor muscles.

The trunk configuration mechanism (TCM) is schematically shown in Figure 13D. This scheme is based on two findings: symmetrical activation of the back muscles with any trunk bending (Fig. 11) and straightening of the trunk with activation of these muscles (Fig. 12B). The symmetrical tonic activity of back muscles secures a straight body axis during unperturbed locomotion (Fig. 13D1). Application of an external force (Fig. 13D2; EF) causes trunk bending in the force direction. The TCM receives sensory input signaling this bending. This input can be provided by stretch receptors of back muscles. It causes strong bilateral activation of back muscles (red lines in Fig. 13D3), which results in an increase of the trunk stiffness (Hu et al., 2009). This increase in stiffness leads to trunk straightening (Fig. 13D4). The straightening occurs with any (left or right) initial trunk bending, suggesting that the direction of motor response is specified not by the motor command but by the trunk shape. The mechanism of trunk straightening could also operate in the intact animal when the forequarters are not fixed but supported by the forelimbs.

We suggest that these four basic mechanisms allow the decerebrate cat to maintain balance during locomotion and to compensate for perturbations considered in the present study (lateral push and stationary lateral tilt of the walkway). Possible integration of these mechanisms in the push task is shown in Figure 13E. We suggest that the same mechanisms operate also in intact animals and contribute to the maintenance of balance during locomotion, by compensating for different perturbations. It should be noted, however, that in intact subjects in environments providing external reference frame for posture to CNS, innate postural mechanisms could be modified, as it was shown in humans (Lackner et al., 2000).

In the present study, we compared the activity of different balance mechanisms when locomotion was evoked by stimulation of MLR or SC, and found no difference (Figs. $5 H, 6 B-E$, $7 B-E)$. Thus, despite some difference in activation of step controlling mechanisms evoked by stimulation of these two sites (Musienko et al., 2012b), the basic postural mechanisms were activated similarly. Specific contribution of spinal and supraspinal mechanisms to the operation of the revealed limb and trunk mechanisms remains unclear.

To conclude, the analysis of balance control during locomotion is a difficult problem due to a number of different mechanisms operating simultaneously. One possible approach to this problem is a computer simulation of the control system, but realistic simulation requires a detailed knowledge about the corresponding neural circuits (Ekeberg and Pearson, 2005; Pearson et al., 2006). With a different approach (using a reduced animal preparation, the decerebrate cat), we managed to analyze separately a number of the mechanisms controlling postural reactions of individual limbs and the trunk. One of the limb mechanisms operates in the transfer phase and secures a standard position for limb landing. Two other limb mechanisms operate in the stance phase; they counteract distortions of the locomotor pattern by regulating the limb stiffness. The trunk configuration mechanism controls the body shape on the basis of sensory information coming from trunk afferents. Postural reactions generated by these four mechanisms are integrated, thus forming a response of the whole system to perturbation of balance during locomotion.

\section{References}

Barbeau H, Rossignol S (1990) The effects of serotonergic drugs on the locomotor pattern and on cutaneous reflexes of the adult chronic spinal cat. Brain Res 514:55-67. CrossRef Medline

Beloozerova IN, Zelenin PV, Popova LB, Orlovsky GN, Grillner S, Deliagina TG (2003) Postural control in the rabbit maintaining balance on the tilting platform. J Neurophysiol 90:3783-3793. CrossRef Medline

Carlson H, Halbertsma J, Zomlefer M (1979) Control of the trunk during walking in the cat. Acta Physiol Scand 105:251-253. CrossRef Medline

Deliagina TG, Zelenin PV, Orlovsky GN (2012) Physiological and circuit mechanisms of postural control. Curr Opin Neurobiol 22:646-652. CrossRef Medline

Duysens J, Clarac F, Cruse H (2000) Load-regulating mechanisms in gait and posture: comparative aspects. Physiol Rev 80:83-133. Medline

Ekeberg O, Pearson K (2005) Computer simulation of stepping in the hind legs of the cat: an examination of mechanisms regulating the stance-toswing transition. J Neurophysiol 94:4256-4268. CrossRef Medline

Garcia-Rill E, Skinner RD (1987a) The mesencephalic locomotor region: I. Activation of a medullary projection site. Brain Res 411:1-12. CrossRef Medline

Garcia-Rill E, Skinner RD (1987b) The mesencephalic locomotor region: II. Projections to reticulospinal neurons. Brain Res 411:13-20. CrossRef Medline

Gerasimenko Y, Musienko P, Bogacheva I, Moshonkina T, Savochin A, Lavrov I, Roy RR, Edgerton VR (2009) Propriospinal bypass of the serotonergic system that can facilitate stepping. J Neurosci 29:5681-5689. CrossRef Medline

Hilliard MJ, Martinez KM, Janssen I, Edwards B, Mille ML, Zhang Y, Rogers MW (2008) Lateral balance factors predict future falls in community- 
living older adults. Arch Phys Med Rehabil 89:1708-1713. CrossRef Medline

Hof AL (1996) Scaling gait data to body size. Gait Posture 4:222-223. CrossRef

Hof AL, Duysens J (2013) Responses of human hip abductor muscles to lateral balance perturbations during walking. Exp Brain Res 230:301-310. CrossRef Medline

Hof AL, Vermerris SM, Gjaltema WA (2010) Balance responses to lateral perturbations in human treadmill walking. J Exp Biol 213:2655-2664. CrossRef Medline

Honeycutt CF, Nichols TR (2010) The decerebrate cat generates the essential features of the force constraint strategy. J Neurophysiol 103:32663273. CrossRef Medline

Honeycutt CF, Gottschall JS, Nichols TR (2009) Electromyographic responses from the hindlimb muscles of the decerebrate cat to horizontal support surface perturbations. J Neurophysiol 101:2751-2761. CrossRef Medline

Hu Y, Wong YL, Lu WW, Kawchuk GN (2009) Creation of an asymmetrical gradient of back muscle activity and spinal stiffness during asymmetrical hip extension. Clin Biomech 24:799-806. CrossRef Medline

Iwahara T, Atsuta Y, Garcia-Rill E, Skinner RD (1992) Spinal cord stimulation-induced locomotion in the adult cat. Brain Res Bull 28:99105. CrossRef Medline

Jordan LM (1986) Initiation of locomotion from the mammalian brainstem. In: Neurobiology of vertebrate locomotion (Grillner S, Stein PSG, Stuart DG, Forssberg H, eds), pp 21-37. London: Macmillan.

Karayannidou A, Zelenin PV, Orlovsky GN, Sirota MG, Beloozerova IN, Deliagina TG (2009a) Maintenance of lateral stability during standing and walking in the cat. J Neurophysiol 101:8-19. CrossRef Medline

Karayannidou A, Beloozerova IN, Zelenin PV, Stout EE, Sirota MG, Orlovsky GN, Deliagina TG (2009b) Activity of pyramidal tract neurons in the cat during standing and walking on an inclined plane. J Physiol 587:37953811. CrossRef Medline

Koehler WJ, Schomburg ED, Steffens H (1984) Phasic modulation of trunk muscle efferents during fictive locomotion in cat. J Physiol 353:187-197. Medline

Kuo A (1999) Stabilization of lateral motion in passive dynamic walking. Int J Robotics Res 18:917-930. CrossRef

Lackner JR, Rabin E, DiZio P (2000) Fingertip contact suppresses the destabilizing influence of leg muscle vibration. J Neurophysiol 84:2217-2224. Medline

Marple-Horvat DE, Amos AJ, Armstrong DM, Criado JM (1993) Changes in the discharge patterns of cat motor cortex neurones during unexpected perturbations of on-going locomotion. J Physiol 462:87-113. Medline

Matsuyama K, Drew T (2000) Vestibulospinal and reticulospinal neuronal activity during locomotion in the intact cat: II. Walking on an inclined plane. J Neurophysiol 84:2257-2276. Medline
McIlroy WE, Maki BE (1999) The control of lateral stability during rapid stepping reactions evoked by anterior-posterior perturbation: does anticipatory control play a role? Gait Posture 9:190-198. CrossRef Medline

Misiaszek JE (2006) Control of frontal plane motion of the hindlimbs in the unrestrained walking cat. J Neurophysiol 96:1816-1828. CrossRef Medline

Musienko PE, Bogacheva IN, Gerasimenko YP (2007) Significance of peripheral feedback in the generation of stepping movements during epidural stimulation of the spinal cord. Neurosci Behav Physiol 37:181-190. CrossRef Medline

Musienko PE, Zelenin PV, Lyalka VF, Orlovsky GN, Deliagina TG (2008) Postural performance in decerebrated rabbit. Behav Brain Res 190:124134. CrossRef Medline

Musienko P, Courtine G, Tibbs JE, Kilimnik V, Savochin A, Garfinkel A, Roy RR, Edgerton VR, Gerasimenko Y (2012a) Somatosensory control of balance during locomotion in decerebrated cat. J Neurophysiol 107: 2072-2082. CrossRef Medline

Musienko PE, Zelenin PV, Lyalka VF, Gerasimenko YP, Orlovsky GN, Deliagina TG (2012b) Spinal and supraspinal control of the direction of stepping during locomotion. J Neurosci 32:17442-17453. CrossRef Medline

Orlovsky GN (1972) The effect of different descending systems on flexor and extensor activity during locomotion. Brain Res 40:359-371. CrossRef Medline

Orlovsky GN, Deliagina TG, Grillner S (1999) Neuronal control of locomotion. In: From mollusc to man. Oxford: Oxford UP.

Pearson K, Ekeberg O, Büschges A (2006) Assessing sensory function in locomotor systems using neuro-mechanical simulations. Trends Neurosci 29:625-631. CrossRef Medline

Pratt CA (1995) Evidence of positive force feedback among hindlimb extensors in the intact standing cat. J Neurophysiol 73:2578-2583. Medline

Reighard J, Jennings HS (1902) Anatomy of the cat. New York: Henry Holf.

Rossignol S, Dubuc R, Gossard JP (2006) Dynamic sensorimotor interactions in locomotion. Physiol Rev 86:89-154. CrossRef Medline

Shik ML, Orlovsky GN (1976) Neurophysiology of locomotor automatism. Physiol Rev 56:465-501. Medline

Shik ML, Severin FV, Orlovskiı GN (1966) Control of walking and running by means of electrical stimulation of the midbrain. Biofizika 11:659-666. Medline

Townsend MA (1985) Biped gait stabilization via foot placement. J Biomech 18:21-38. Medline

Zelenin PV, Musienko PE, Gerasimenko YP, Orlovsky GN, Deliagina TG (2013) Mechanisms of balance control during locomotion. Soc Neurosci Abstr 39:832.06.

Zomlefer MR, Provencher J, Blanchette G, Rossignol S (1984) Electromyographic study of lumgbar back muscles during locomotion in acute high decerebrate and in low spinal cats. Brain Res 290:249-260. CrossRef Medline 